\title{
Strain-injection and crack-path field techniques for 3D crack-propagation modelling in quasi-brittle materials
}

\author{
I. F. Dias ${ }^{1, a}$, J. Oliver ${ }^{2,3, b}$ and O. Lloberas-Valls ${ }^{2,3, c}$ \\ ${ }^{1}$ Laboratório Nacional de Engenharia Civil (LNEC) \\ Avenida Brasil 101,1700 Lisboa, Portugal \\ ${ }^{2}$ E.T.S. d'Enginyers de Camins, Canals i Ports, Technical University of Catalonia \\ ${ }^{3} \mathrm{CIMNE}$ - Centre Internacional de Metodes Numerics en Enginyeria \\ Campus Nord UPC, Edifici C-1, c/Jordi Girona 1-3, 08034 Barcelona, Spain. \\ aidias@Inec.pt, ${ }^{b}$ xavier.oliver@upc.edu, ${ }^{c}$ olloberas@cimne.upc.edu
}

Keywords: computational material failure, strong discontinuities, crack-path field, strain injection, mixed formulations, strain localization, crack propagation

\begin{abstract}
This paper presents a finite element approach for modelling three-dimensional crack propagation in quasi-brittle materials, based on the strain injection and the crack-path field techniques. These numerical techniques were already tested and validated by static and dynamic simulations in 2D classical benchmarks [1-3] and, also, for modelling tensile crack propagation in real concrete structures, like concrete gravity dams [4]. The main advantages of the methodology are the low computational cost and the independence of the results on the size and orientation of the finite element mesh. These advantages were highlighted in previous works by the authors and motivate the present extension to $3 \mathrm{D}$ cases.
\end{abstract}

The proposed methodology is implemented in the finite element framework using continuum constitutive models equipped with strain softening and consists, essentially, in injecting the elements candidate to capture the cracks with some goal oriented strain modes for improving the performance of the injected elements for simulating propagating displacement discontinuities. The goal-oriented strain modes are introduced by resorting to mixed formulations and to the Continuum Strong Discontinuity Approach (CSDA), while the crack position inside the finite elements is retrieved by resorting to the crack-path field technique.

Representative numerical simulations in 3D benchmarks show that the advantages of the methodology already pointed out in 2D are kept in 3D scenarios.

\section{Introduction}

Computational failure mechanics is a field of research that has attracted significant interest in the last decades. The safety of the engineering designs motivates the research in the field. Numerical methods that can realistically simulate crack propagation have become relevant tools for modeling failure scenarios. They allow a safe and efficient design of new structures, quantification of safety factors of the existing ones, in front of non-expected loads, and the assessment of the remaining structural life of structures that, due to ageing and degradation of the constituent materials, have lost part of their initial load bearing capacity. 
Within the context of the finite element method, the earliest methodologies for modeling propagating cracks have shown to provide mesh dependent results [5,6]. A systematic analysis of the main numerical problems arising from these methodologies, stress locking and mesh-bias dependence, was first done in [7]. These numerical pathologies have motivated a subsequent huge scientific research on the field, and a multiplicity of methods have been proposed and developed by different authors.

One important family of methods is based on the regularization of standard continua equipped with strain-softening. These methodologies incorporate a characteristic length and the discontinuity is captured by a band with bandwidth, $h$, encompassing several finite elements. For this reason, this family of methods can be also referred in [2] as supra-element-band methods. Non local [8-10] and gradientenriched $[11,12]$ models, which share some similarities with the more recently proposed phase-field models of fracture $[13,14]$, can be included in this category.

Another relevant family of methods considers the discontinuity captured inside the finite element. In these methodologies, also termed intra-element methods [2], the finite elements are enriched with additional discontinuous displacement modes and they give rise to the E-FEM [15-17] or X-FEM techniques $[18,19]$ when these additional modes have an elemental or nodal support, respectively.

Despite the huge advances that have been achieved in the last years, most of the existing methods have shown limitations for being applied in modelling real life problems, either because the models have been implemented only for 2D cases, or because the computational cost of the 3D implementation is unaffordable when applied to lange structures. In fact, many times
parallel, computing equipment, not available to most of the researcl
The remarkable properties of the strain injection techniques in ter
computational cost, pointed out in 2D modelling, either for static[ $20]^{1}$, have motivated the authors to perform the pr

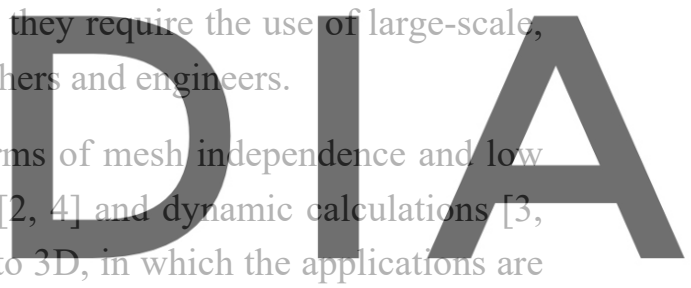
computationally much more demanding. Therefore, the main goal of this work is to provide evidence for free at https//WwW.Scipedia.com to download the version without the watermark that the methodology can be extended to three dimensions, while retaining its main advantages, so a mesh independent time-affordable methodology, which can be used in a standard personal computer for modelling 3D crack propagation and material failure, can be proposed and validated.

In order to validate the technology, some applications to 3D benchmarks have been performed and results are compared with experimental or numerical tests by other authors. Since the goal of the paper is focused on the 3D extension, the inertial effects are left aside and the kinematical description of the motion is simplified to infinitesimal strains.

The finite element formulation presented in this work closely follows the formulation derived in the previous publication [4], where a mixed stabilized formulation is used for all the domain. In order to provide the unfamiliar reader with the main features of the methodology, a brief theoretical summary is given in sections 2, 3 and 4, but specific details, that can be consulted in [1-4], are not included for the sake of length and avoiding repetition of material already published.

\footnotetext{
${ }^{1}$ For dynamic cases remarkably coarse meshes were used when compared with competing methods, as pointed out in [3].
} 


\section{Adopted variational 3D approach to the mechanical problem}

Standard finite element formulations of the non-linear mechanical problem (summarized in Box 1) are usually displacement-based, in the sense that the displacement field, $\boldsymbol{u}(\mathbf{x}, t)$, is the independent unknown to be interpolated by means of suitable interpolation functions. Due to implementation reasons, the mechanical formulation will be presented in rate form.

Given the external actions, $\boldsymbol{w}_{\mathbb{*}}^{*}(\mathbf{x}, t), \mathbb{E}^{\mathbb{E}}(\mathbf{x}, t)$ and $\left.\mathbf{b} \& \mathbf{x}, t\right)$, find $\left.\boldsymbol{u} \&(\mathbf{x}, t), \& \& \mathbf{x}, t\right)$ and $s \&(\mathbf{x}, t)$ :

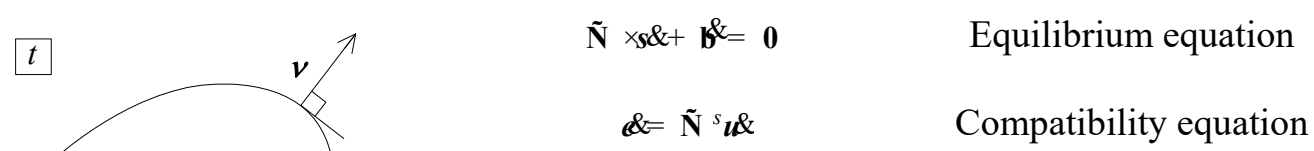

$\&=\tilde{\mathbf{N}}^{s} \boldsymbol{u} \quad$ Compatibility equation

B

$\boldsymbol{s} \&=\boldsymbol{S} \&(\& a, a) \dot{\mathrm{j}}$
$a \&=g(\boldsymbol{e}, a)$

Constitutive equation

Internal variables

evolution

Boundary conditions

Box 1: Mechanical problem in the body B. Herein $\mathscr{\vartheta}_{u} \mathrm{~B}$ and $\boldsymbol{\vartheta}_{s} \mathrm{~B}$ are the portions of the boundary $\boldsymbol{q} \mathrm{B}$ where Dirichlet and Newman conditions are defined, $n$ is its outward normal and $u \mathbb{E}^{*}(\mathbf{x}, t)$, \& $\mathbb{E}^{\mathbb{E}}(\mathbf{x}, t)$ and $\left.\mathbf{b} \& \mathbf{x}, t\right)$

are the prescribed displacements, tractions and body loads, respectively. In equation (3) $s, e, a$ stand,

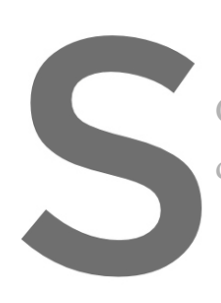

respectively for the stresses, the strains and
Conversely, this work uses the mixed stabilized
displacements and strains $u / e$ :
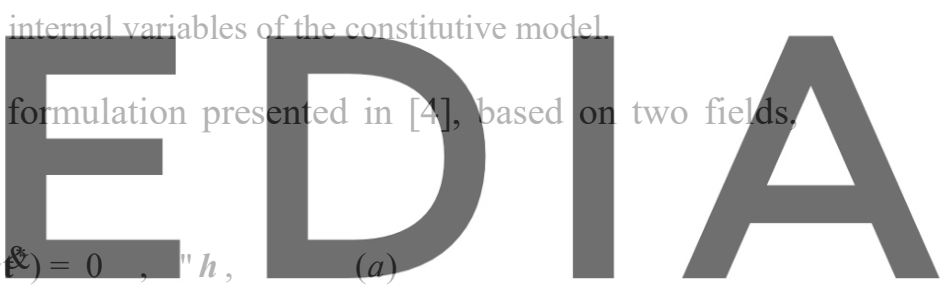

(5)

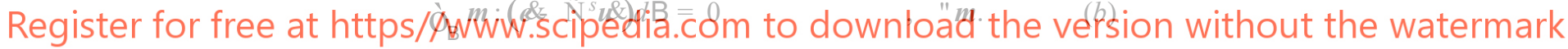

where equations (5)(a) and (5)(b) correspond to weak forms of the equilibrium (1) and compatibility

(2) equations, respectively, $h^{\& e x t}$ stands for the work rate produced by the external forces and $\boldsymbol{h}$ and $\boldsymbol{m}$ for suitable test functions. In comparison with the one-field case, the mixed formulation in equation (5) renders a more flexible approach for modelling material failure, since it gives additional freedom in the choice of the strain interpolation.

By choosing standard bi-linear interpolation functions for the displacement field and element-wise constant interpolation for the strains, in eight-noded hexahedral finite elements, and adding a consistent stabilizing term to the equations, the finite element approximation of equation (5) reads, after some mathematical manipulation (see [4]):

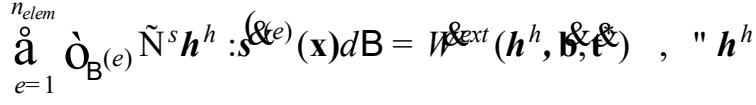

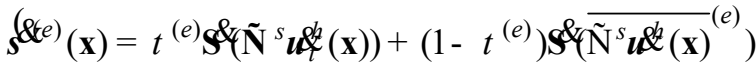


where $s^{(\& 6)}$ are the elemental stabilized stresses, $s^{\&}$ is the non-linear constitutive function returning the stresses from the strains, $t^{(e)}$ is the stabilization factor, taken in this work equal to 0.1 , and $\overline{\tilde{\mathrm{N}}^{s} \mathbf{u}^{2}(\mathbf{x})}(e)$ stands for the mean value of the symmetric gradient of the displacements over the finite element.

This mixed formulation is particularly suited for developing the strain injection techniques since the strain modes to be injected are also proposed by resorting to mixed $\boldsymbol{u} / \boldsymbol{e}$ finite element approaches.

\section{Strain injection techniques}

The main idea of the strain injection technique consists of improving the performance of some specific elements (belonging to the injection domain, $\mathrm{B}_{i n j}$ ) for modeling strain localization by injecting specific enhanced strain modes, while the remaining elements keep using the base formulation (in the present work the mixed formulation (6)) typically in the zones where the body behaves elastically (Figure 1).

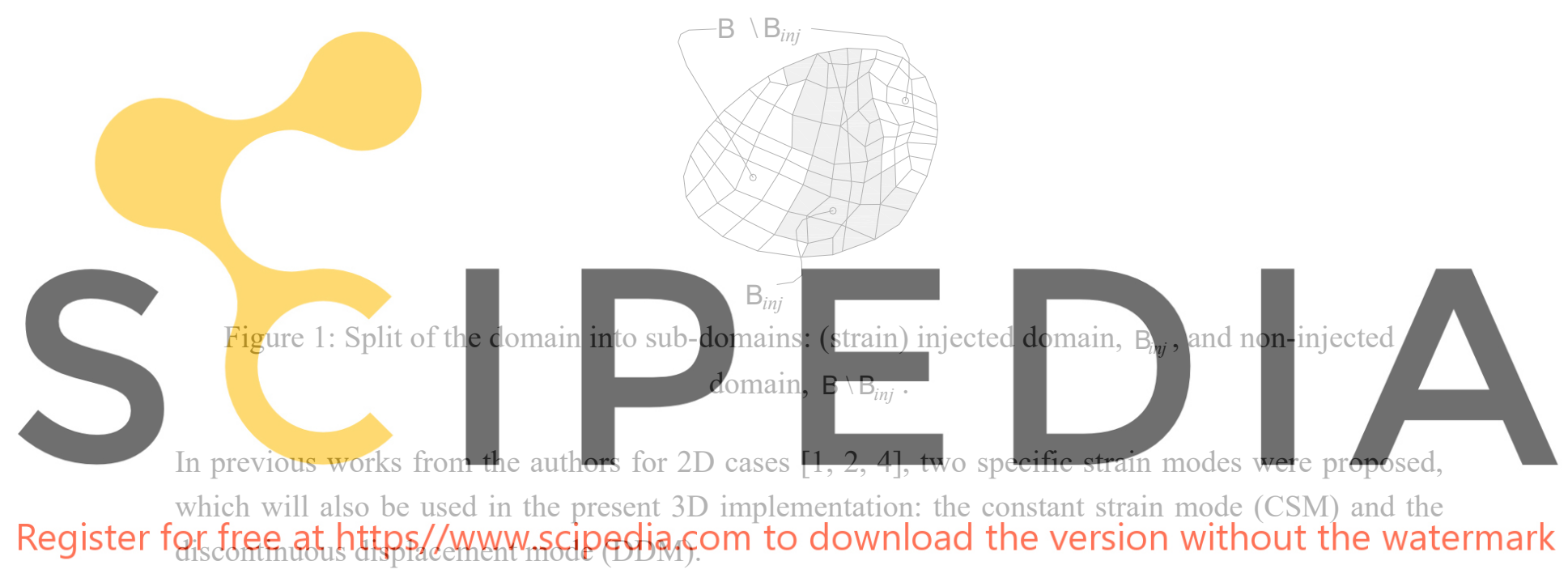

The CSM will be injected in those elements that initially verify the bifurcation condition ([21]), which, therefore, are regarded as "candidates" for developing displacement discontinuities. We would like to emphasize that not all of these "candidates" elements, will develop discontinuities at this, or subsequent times. In fact as the loading process goes on, strains tend to localize in narrow bands, so the elements outside this band will naturally unload, leaving, therefore, the injection domain and recovering the mixed finite element formulation (6). On the other hand, those elements where strains remain localizing will be reached by the evolving crack path (see section 5), so they will be injected with the discontinuity displacement mode (DDM), for enriching their ability to capture accurately the crack opening inside the finite elements, without spurious stress transfer to the neighboring elements. Figure 2 illustrates the process. 

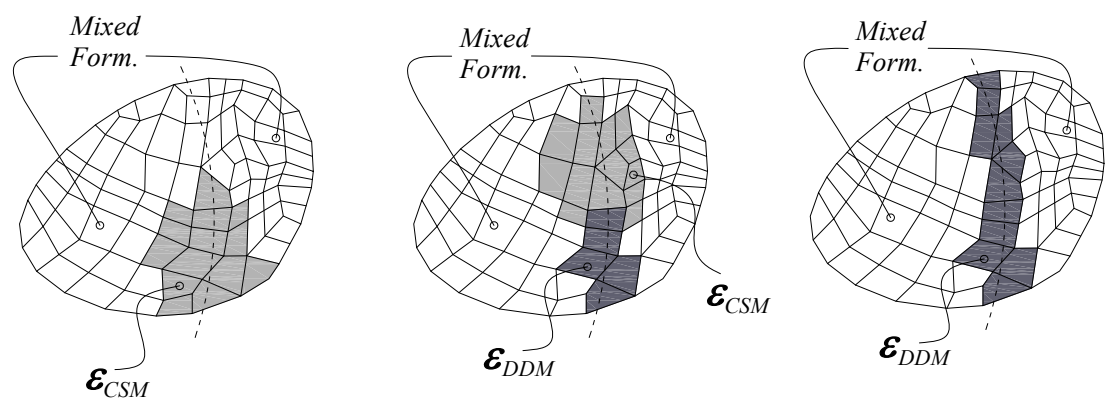

Figure 2: Typical evolution of the injection domains along time. In light gray are identified the elements injected with the constant strain mode $\left(\boldsymbol{e}_{C S M}\right)$ while in dark gray are identified de elements injected with the discontinuous displacement mode $\left(\boldsymbol{e}_{D D M}\right)$.

The CSM injection is obtained by considering the strains constant inside the finite element. Here it is highlighted the convenience of the mixed finite element formulation, since this mode can be activated just by considering $t^{(e)}=0$ in equation (6), which renders:

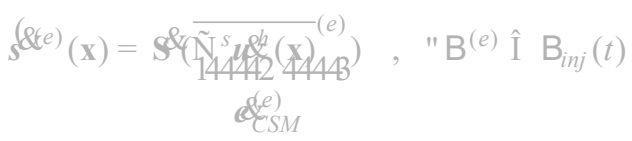
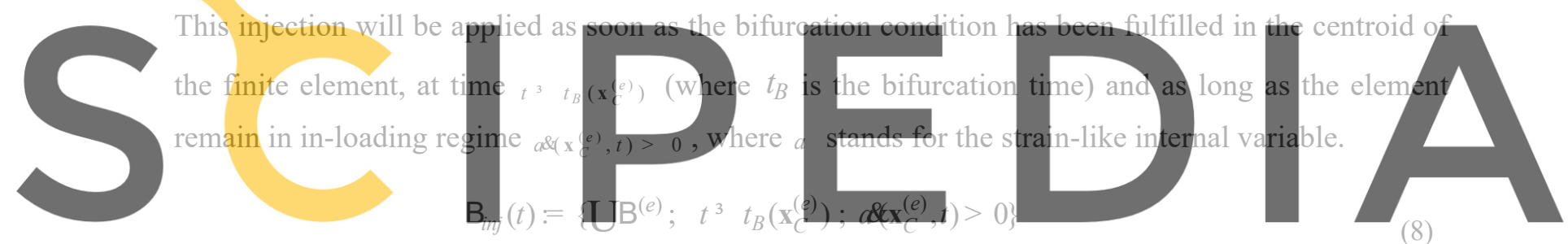

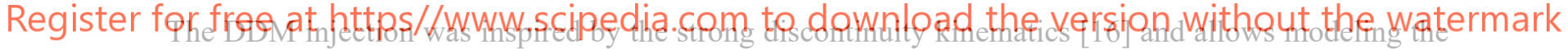

crack opening behavior inside the finite elements without stress locking defects. After some

manipulations that can be consulted in previous works of the authors [1,2], the discontinuous displacement mode can be written as:

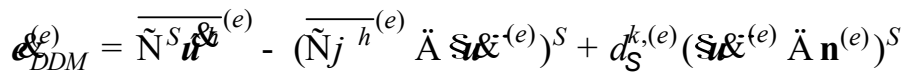

where $\overline{(\cdot)}$ stands for the mean value of (.) over the element, $\mathrm{S}$ is the discontinuity surface, $\mathbf{n}$ is the unit vector orthogonal to $\mathrm{S}$, if is the smooth part of the displacement field, sid " is the (rate of) displacement jump inside the finite element, $j(\mathbf{x})$ is a continuous indicator function (see $[1,2]$ ), taking values of 0 or 1 for each side of the discontinuity, $d_{S}$ is the Dirac distribution shifted to $\mathrm{S}$ and $k$ is the (small) regularization parameter, used, for computational purposes, to regularize the Dirac's generalized function and standing for the bandwidth of the band capturing the jump. The regularizing parameter, $k$, can be defined as a fraction of the elemental effective bandwidth $1^{(e)}$ computed as:

$$
1^{(e)}=\frac{\operatorname{meas}\left(\mathrm{B}^{(e)}\right)}{\operatorname{meas}\left(\mathrm{S}^{(e)}\right)} \quad, k=x l^{(e)}
$$

where $x$ can take values in the semi-open interval $(0,1.0]$. The semi-open character of the interval around 0 is limited by the computer accuracy.

The DDM injection will be applied when conditions in (8) are fulfilled and, additionally, when the element is identified as capturing a discontinuity. For that, the next two conditions have to be satisfied: 
1- The elements are crossed by the discontinuity path $G_{t}$. In order to inject the DDM, the progression of the actual discontinuity path should be identified a little in advance. In the context of the strain injection techniques this is obtained by the crack path field technique described in Section 4.

2- The barycenter of the finite element has achieved a "sufficient" degree of softening. This is imposed in terms of the stress-like internal variable $q\left(\mathbf{x}_{C}^{(e)}, t\right)$, as following:

$$
q\left(\mathbf{x}_{C}^{(e)}, t\right) £ g q_{b i f}\left(\mathbf{x}_{C}^{(e)}\right)
$$

where $q_{b i f}\left(\mathbf{x}_{C}^{(e)}\right)$ is the value of internal variable at the bifurcation time and $g$ is a user-parameter defining the injection threshold (typically $g \hat{I}[0.9,1.0]$ ).

REMARK 3-1 The methodology used in this work is a combination of the strain injection and the crack path field techniques in Section 4. In fact, for effectively injecting the DDM, the crack path should be identified in advance. For ensuring that the crack path is well captured a delay between the DDM injection and the bifurcation time has to be considered. The user-defined parameter $g$ in equation (11) plays this role, safeguarding that a "sufficient" degree of softening was achieved prior to the injection. From the author's experience, $g=0.95$ provides a good balance between the (little) error produced by delaying
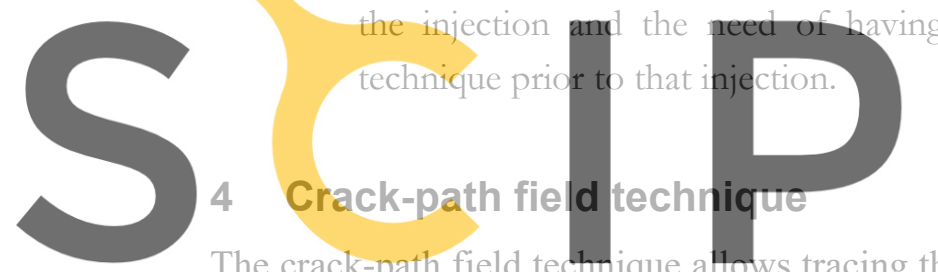

The crack-path field techrique allows tracing the
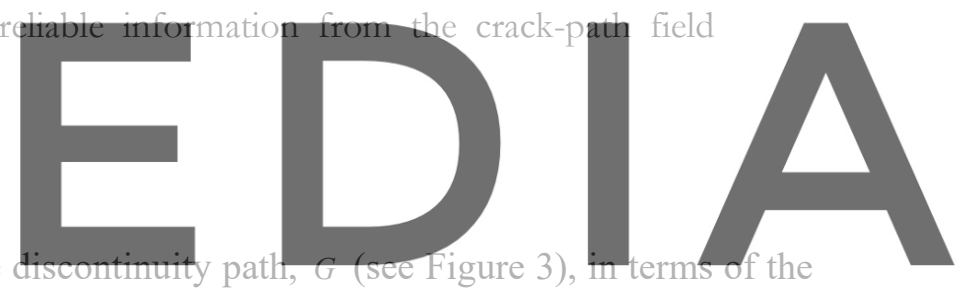

strain localization pattern that arises, naturally, by introducing softening in the constitutive relations Register for free at https//www.scipedia com to download the version without the watermark

compute the relative maxima of a, conveniently chosen, localizing field. In this work, we chose to use for such purpose the strain like internal variable $a^{h}$ of the continuum constitutive model (Figure 3). In practice, the locus of relative maxima of the localizing field can be computed by means of the zero level-set of its normal directional derivative (see Figure 4). 


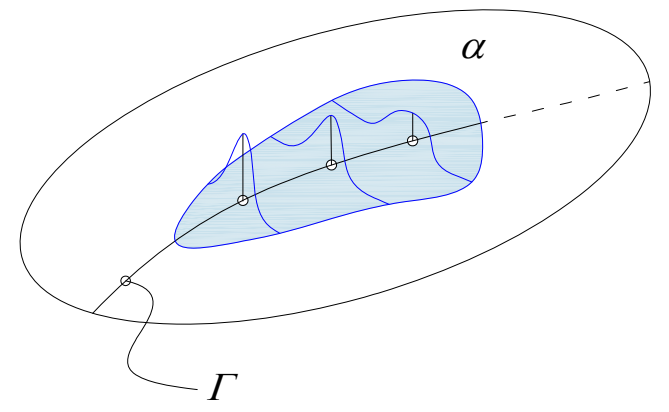

Figure 3: Schematic distribution of a localizing strain-like internal variable, $a$, in a 2D problem.

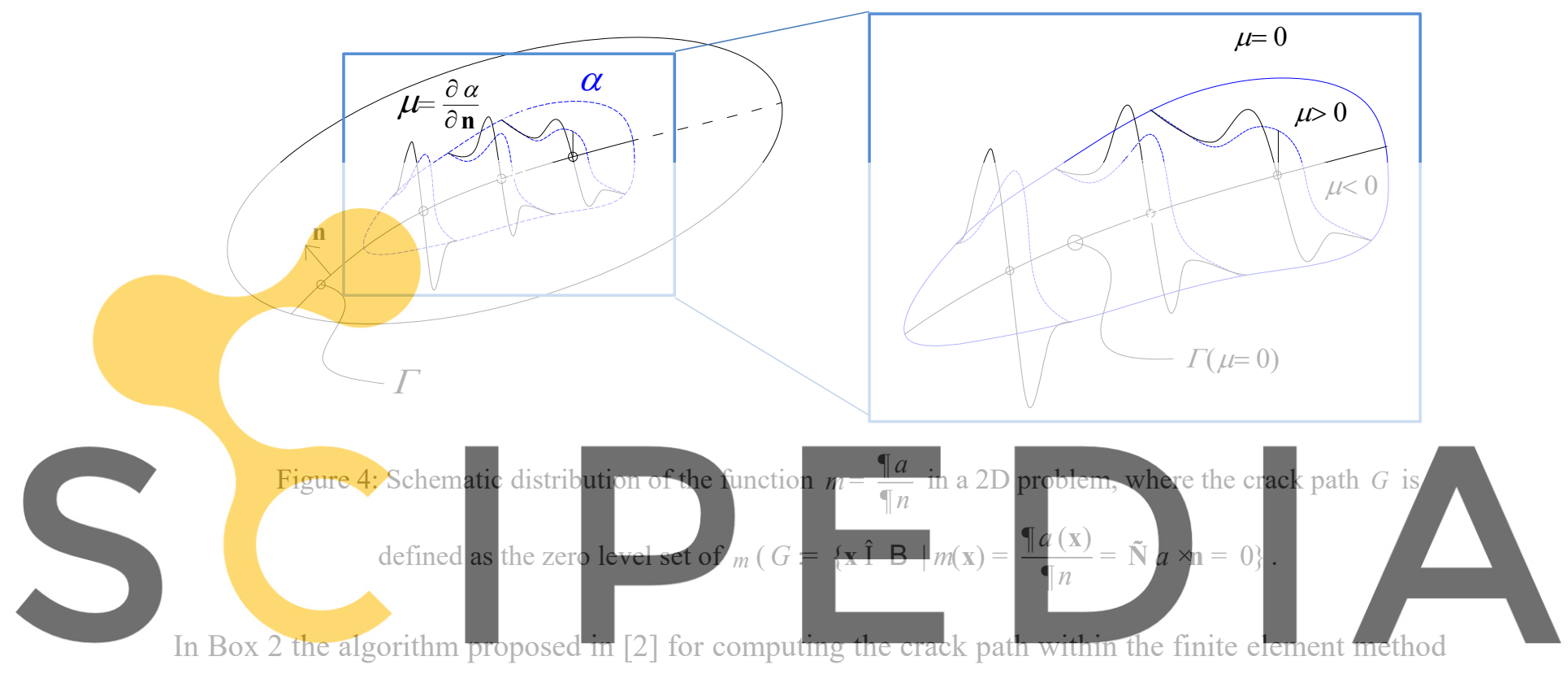

Register for free at https//www.scipedia.com to download the version without the watermark

1 - Compute $y^{h}$ as a smoothed continuous approximation of the localizing variable $a^{h}$ :

$$
\grave{\mathrm{O}}_{\mathrm{B}^{h}} \bar{y}^{h}\left(y_{t}^{h}-a^{h}(\mathbf{x}, t) d \mathrm{~B}=0, " \bar{y}^{h}\right.
$$

2- Compute the derivative in the direction orthogonal to the discontinuity, $\mathbf{n}$ :

$$
\frac{\mathbf{I} y_{t}^{h}}{\mathbb{\Phi} n}=\tilde{\mathbf{N}} y_{t}^{h} \times \mathbf{n}
$$

3- Compute the crack-path-field $m^{h}$ as a smooth continuous approximation of that directional derivative:

$$
\grave{\mathrm{O}}_{\mathrm{B}^{h}} \bar{m}^{h}\left(m_{t}^{h}-\tilde{\mathbf{N}} y_{t}^{h} \times \mathbf{n}\right) d \mathrm{~B}=0, \quad " \bar{m}^{h}
$$

4- Determine the crack-path position, $G_{t}$, as the zero level set of $m_{t}^{h}$ in an elemental basis:

$$
G_{t}^{h}:=\left\{\mathbf{x} \hat{I} \quad B^{h} ; m_{t}^{h}(\mathbf{x})=0\right\}{ }^{\circledR} \text { zero level set of } m_{t}^{h}(\mathbf{x})
$$

Box 2: Summary of the Crack-path-field problem.

For $1 \mathrm{D}$ cases the crack path $G_{t}$ corresponds to a point, for $2 \mathrm{D}$ to a line and in the present $3 \mathrm{D}$ implementation corresponds to a surface. We would like to remark that once the crack-path-field $m_{t}^{h}(\mathbf{x})$ 
is computed at the domain nodes, the exact position of the crack path thought the element can be easily retrieved in an element basis, in terms of its nodal values (Figure 5).
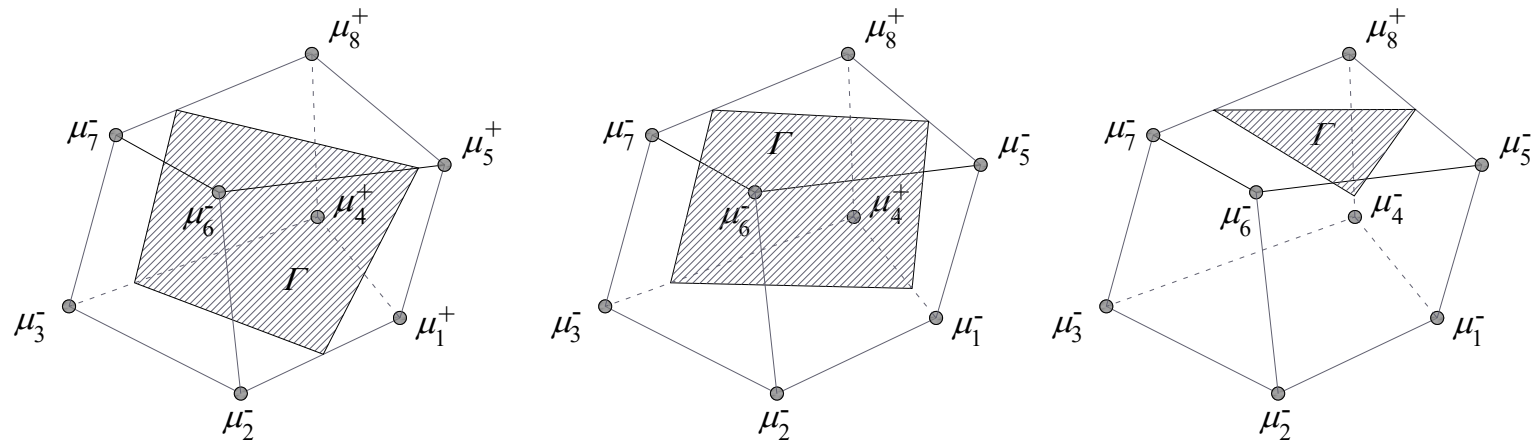

Figure 5: Retrieving the elemental positon of the crack from the nodal crack-path-field values: sign changing from positive, $\left(m_{i}^{+}\right)$, to negative, $\left(m_{j}\right)$, identifies that the elemental edge, $(i-j)$, is crossed by the crack (the crossing point is identified for $m(\mathbf{x})=0$ assuming a linear evolution the edge). Potential situations for linear hexahedral elements are sketched in the figure.

\section{Representative numerical simulations}

In order to asses the performance of the strain injection and the crack-path field techniques proposed in

this work, a number of

are presented in the followi

5.1 Homogeneous strip subjected to damage model
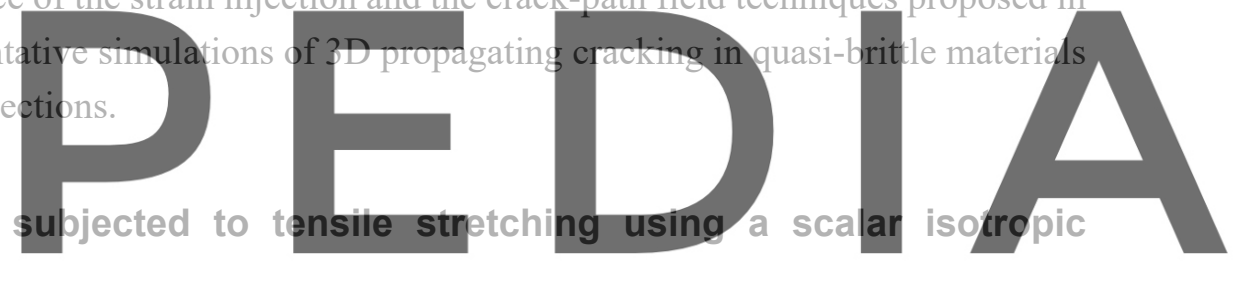

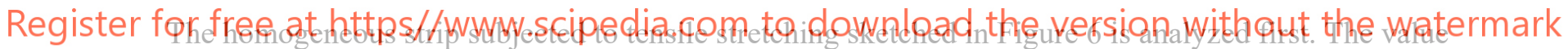

of this simple problem comes from the fact that an exact analytical solution is available, so the results provided by the numerical simulation can be rigorously compared to an exact solution. This example was already analyzed by the authors in a previous work [2] in 2D (where the strip was idealized in 2D plane strain conditions), and is used here as a first step for validating the $3 \mathrm{D}$ implementation.

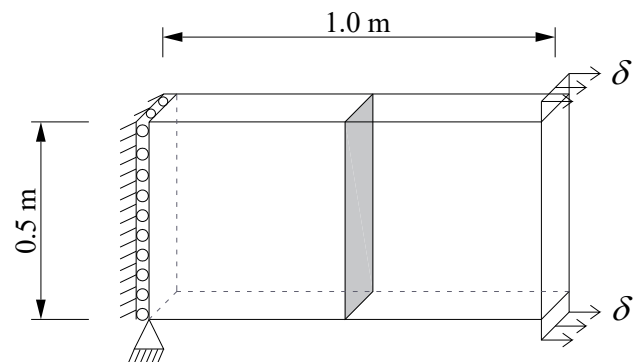

\begin{tabular}{cccc}
\hline $\begin{array}{c}\sigma_{u} \\
{[\mathrm{kPa}]}\end{array}$ & $\begin{array}{c}E \\
{[\mathrm{kPa}]}\end{array}$ & $v$ & $\begin{array}{c}G_{f} \\
{[\mathrm{~N} / \mathrm{m}]}\end{array}$ \\
\hline 2.0 & 100 & 0.0 & 22.7 \\
\hline
\end{tabular}

Figure 6: Homogeneous strip subjected to tensile stretching. Geometry $(\mathrm{m})$ and material parameters, being $s_{u}$ the ultimate stress, $E$ the Young's modulus, $n$ the Poisson's ratio, $G_{f}$ the fracture energy and $g$ is the user-parameter defining the DDM injection threshold (see REMARK 3-1).

The theoretical solution for this quasi-homogeneous problem, when using a scalar isotropic damage model (slightly perturbed at some point to break the initial homogeneity) with null Poisson ratio, consists of a vertical crack passing through the perturbed point. In order to challenge the methodology, 
a miss-oriented mesh of 8-node hexahedral finite elements was used to perform the numerical tests, as shown in Figure 7, where results obtained with the standard displacement based formulation and the strain injection techniques are plotted. As it can be observed, the results obtained with the standard formulation are clearly affected by the mesh bias, since the localization band follows de mesh alignment and a spurious tangential displacement jump is obtained. On the other hand, when using the strain injection techniques the localization band "zigzags" through the mesh and, in spite of the challenge posed by the mesh bias, a vertical crack pattern is obtained consisting in a crack opening in mode I of fracture (with zero tangential displacement jump) (see Figure 8). These results, along with the force displacement curves depicted in Figure 9, provide strong evidence of the advantages and accuracy of the strain injection techniques for modeling crack propagation in $3 \mathrm{D}$ problems.

a)
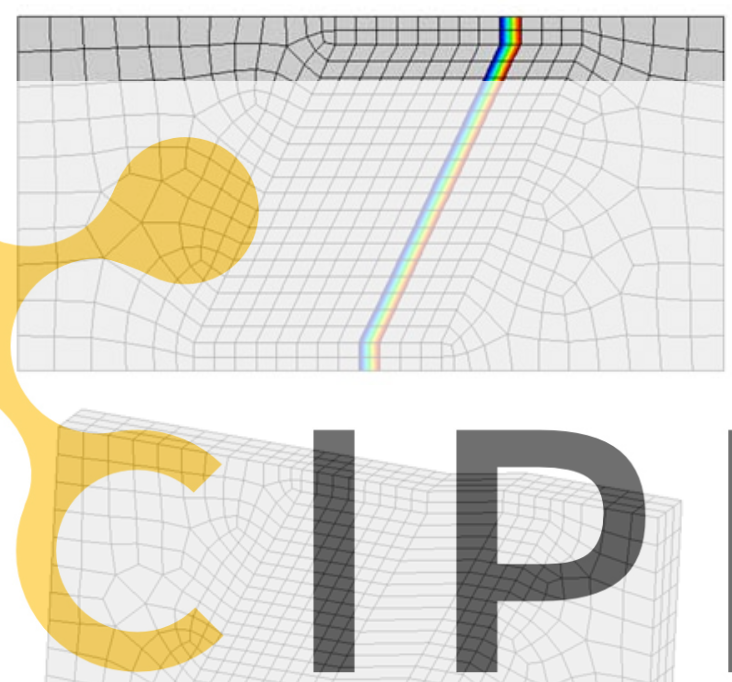

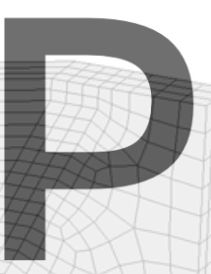

b)
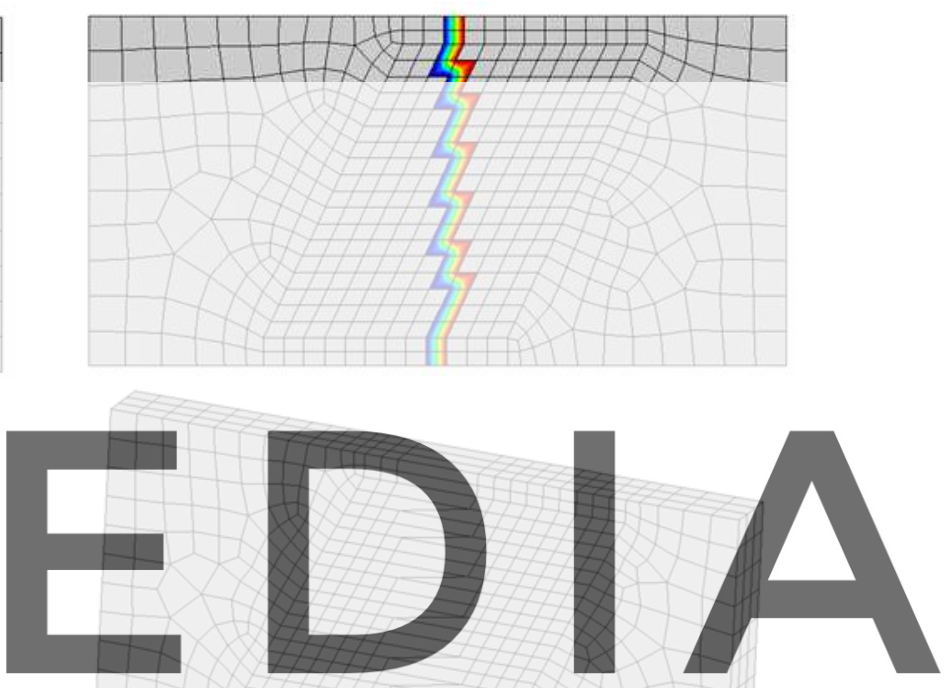

Register for free at https//www.scipedia.com to download the version without the watermark

Figure 7: Strip subjected to tensile stretching. Iso-displacement contours and deformed meshes. Results obtained with: a) Irreducible displacement based formulation and b) strain injection techniques.

The finite element mesh has a total of 1464 finite elements and 6336 degrees of freedom.

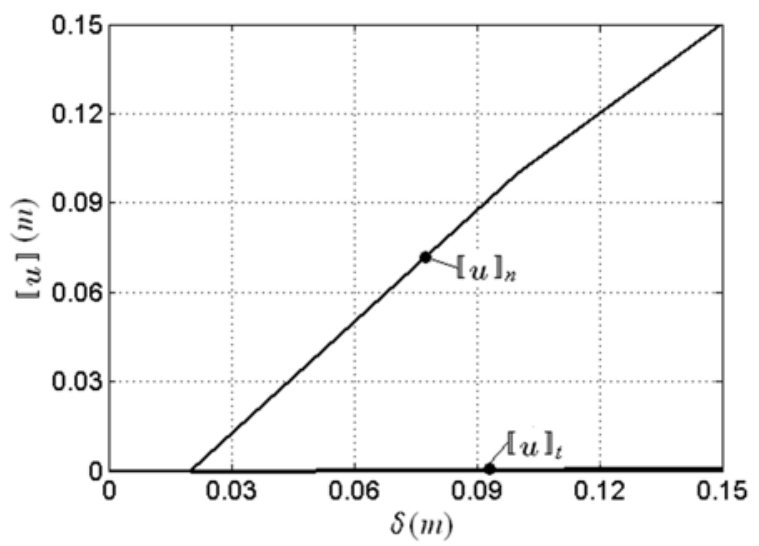

Figure 8: Strip subjected to tensile stretching. Displacement jump evolution (normal $\mathfrak{s}_{\boldsymbol{i}} \bar{n}_{n}$ and tangential \$i $\ddot{t}_{t}$ components. Results computed by using the strain injection technique. 


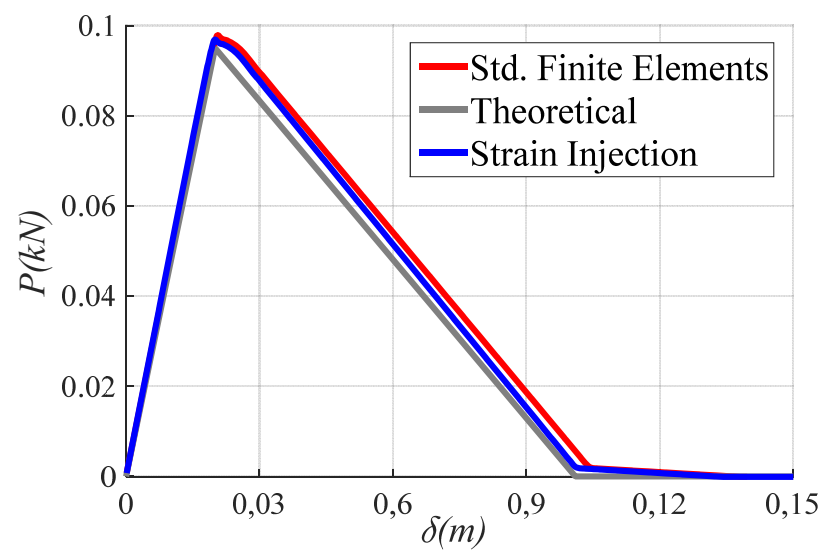

Figure 9: Strip subjected to tensile stretching. Reaction force vs.displacement curves.
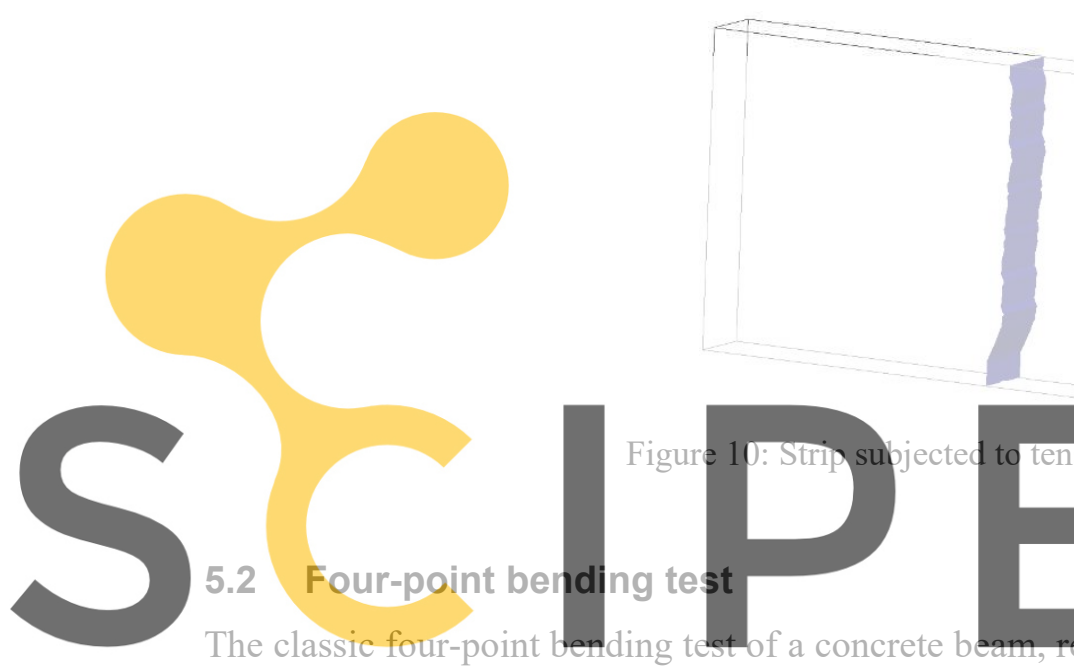

as a benchmark by various authors (e.g. [7, 23-26]), is now considered by using a Rankine-type

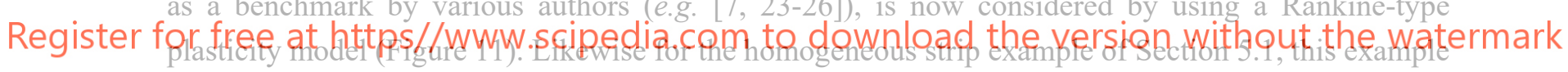

was previously analysed by the authors in [2] (where the 3D geometry was simplified to ideal 2D plane stress conditions). Here it is used as second validation step of the 3D implementation, now by considering a bending beam.

The results reported by using physical experiments, consist of a curved fracture propagating from the notch tip, as it is depicted in Figure 11. The numerical analysis was carried out by controlling the Crack Mouth Sliding Displacement (CMSD) at the notch. 


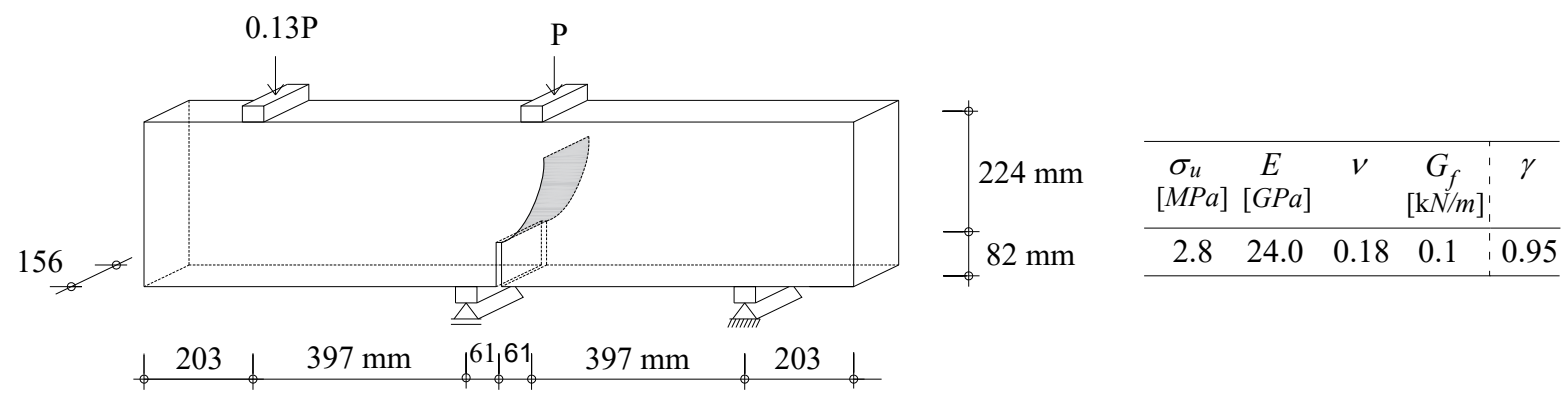

Figure 11: Four-point bending test. Geometry $(\mathrm{mm})$ and material parameters, being $s_{u}$ the ultimate stress, $E$ the Young's modulus, $n$ the Poisson's ratio, $G_{f}$ the fracture energy and $g$ is the user-parameter defining the DDM injection threshold (see REMARK 3-1).

Figure 12 shows some results obtained with the strain injection techniques using an unstructured mesh. It can be observed that the crack trajectory compares well with the experimental result. The plot of the iso-displacement contours along with the deformed mesh shows the sharpness of the strain localization band, that encompasses just one finite element, this indicating that stress-locking effects are effectively circumvented. 
a)

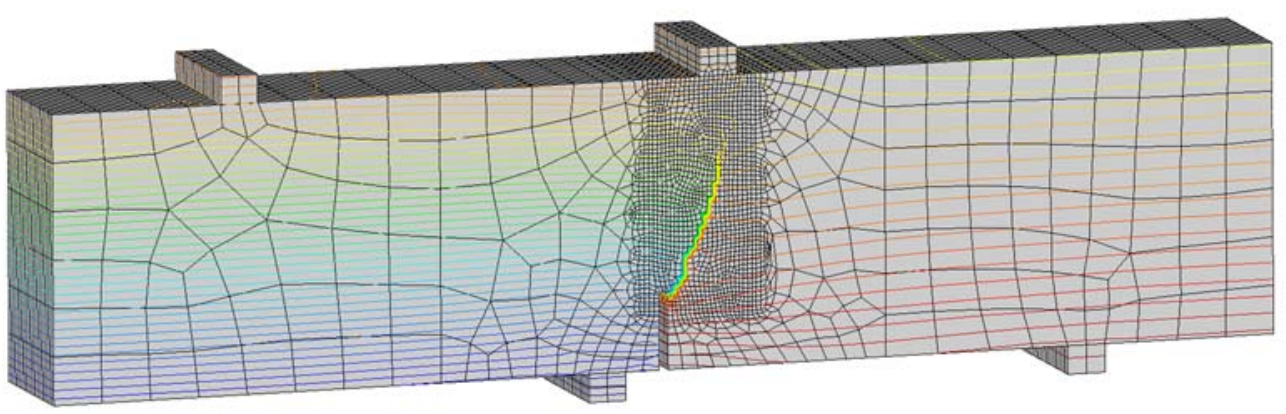

b)

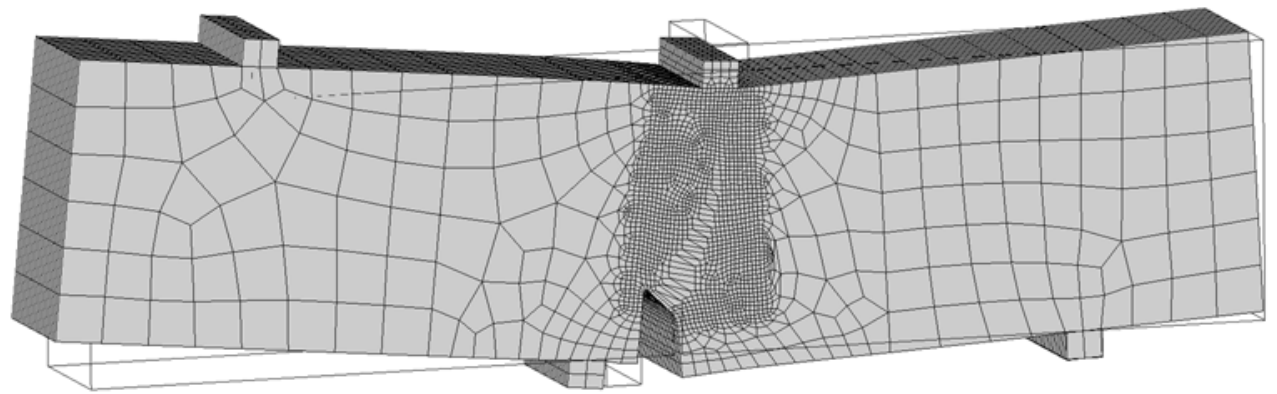

c)

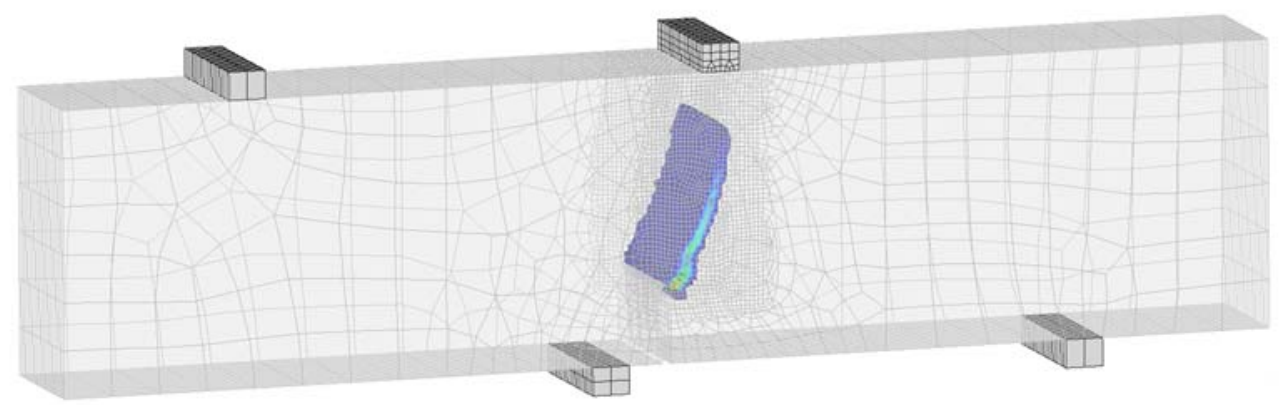

Figure 12: Four-point bending test. Results obtained with the strain injection techniques: a) Isodisplacement contours; b) deformed mesh and c) iso-surfaces of the equivalent plastic deformation (smothed). The finite element mesh has a total of 25600 hexahedral finite elements and 83916 degrees of freedom.

Figure 13 shows the evolution of the injection domains for three stages of the analysis, where stage c) corresponds to the end of the analysis. Figure 14 displays, at stage c), the crack path computed inside de elements injected with the DDM mode. 
a)

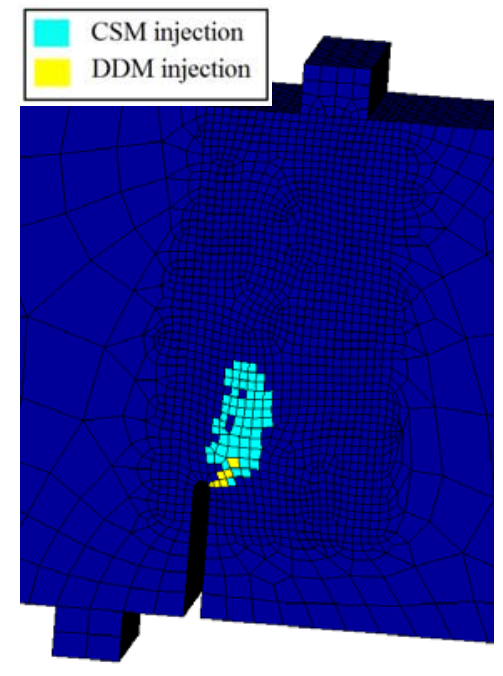

b)

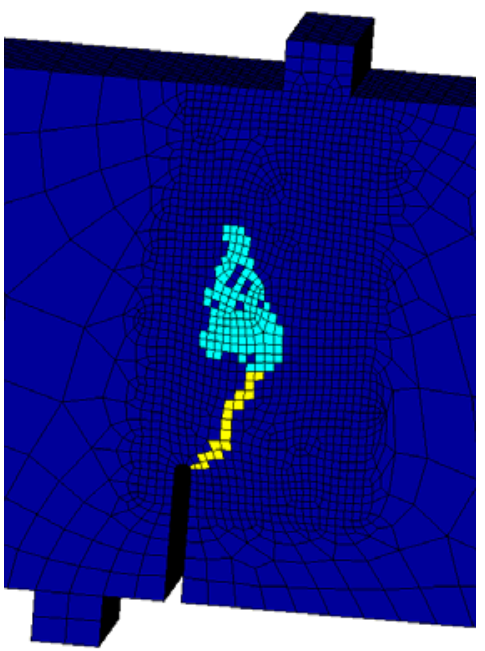

c)

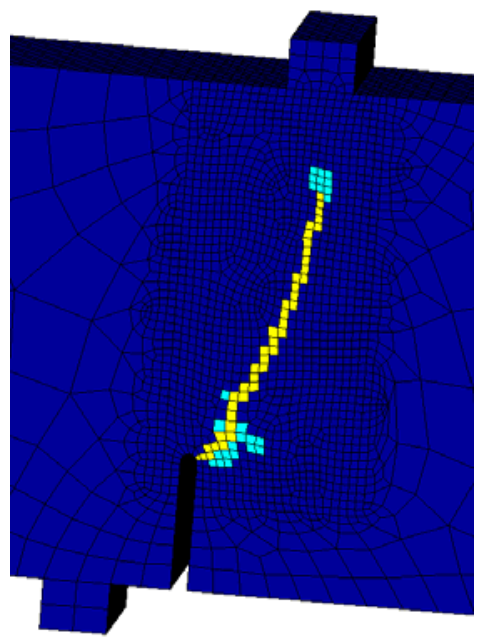

Figure 13: Four-point bending test. Evolution of the injection domains for different stages of analysis.

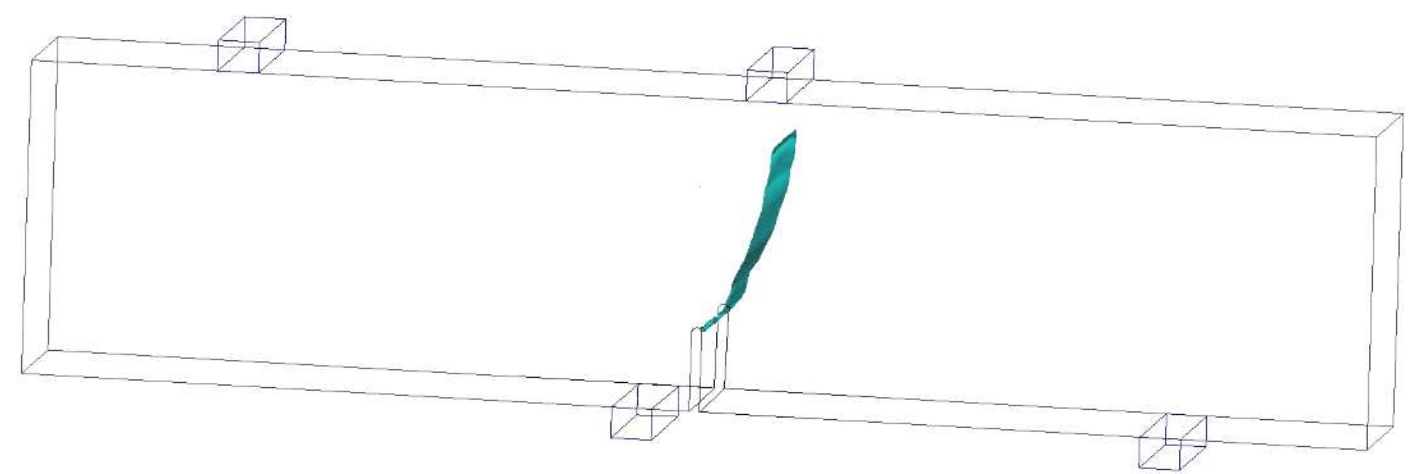

Figure 14: Four-point bending test. Crack path computed inside de elements injected with the DDM mode.

For a quantitative comparison, the force-displacement curves obtained by using the strain injection techniques and the standard displacement based finite element formulation are plotted in Figure 15. In the figure the experimental envelop provided in [22] (shaded zone) is also depicted. The plot shows that the results obtained with the standard formulation are much more dissipative (area below de curve) than the ones obtained by the strain injection, that fit well in the experimental envelop. This fact gives additional evidence that stress locking is effectively minimized by the proposed injection techniques. 


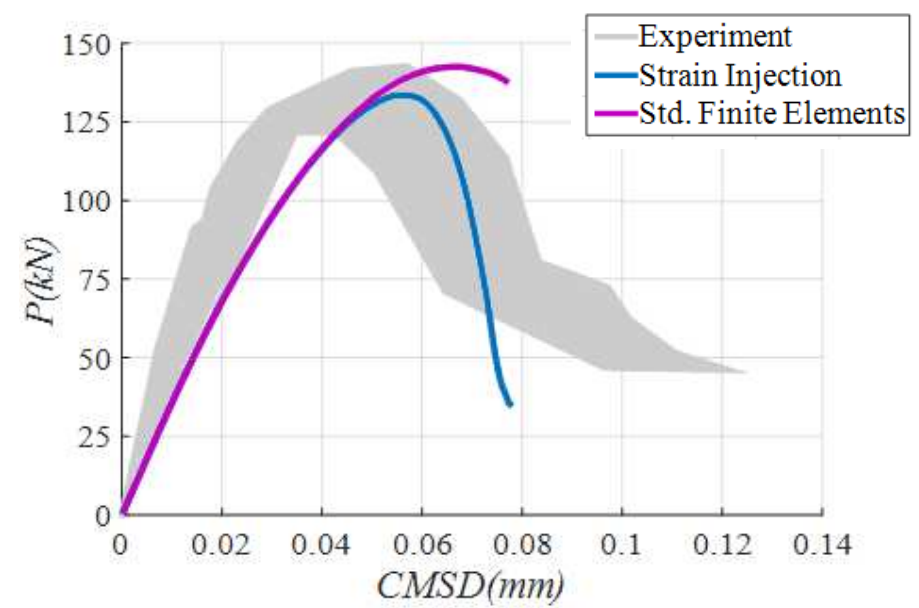

Figure 15: Four-point bending test. Force displacement curves.

\begin{abstract}
REMARK 5-1. It is known in the Continuum Strong Discontinuity Approach that when strong discontinuities are used with Rankine-type plasticity models, the so-called strong discontinuity conditions place restrictions on the displacement jump [27], that has to evolve in pure mode I (without tangential component). In case of curved cracks these conditions constrain the crack to evolve because cracks in pure mode I have to propagate in a straight path. This incompatibility was analysed in [2] and termed as kinematic locking. In order to alleviate this kinematic restriction, the regularization parameter $k$, of equation (10), was set, for this example, equal to the element size $1^{(e)}$, this allowing a mixed mode compatible with the curved crack to propagate without locking effects.
\end{abstract}

\title{
5.3 Pull-out test
}

This example consists of the numerical simulation of the pullout of a steel anchor embedded in a concrete block. Pullout tests have been investigated, either numerically or experimentally, by diverse authors using a variety of experimental setups. The first numerical studies have been performed in two dimensions using axisymmetric plane finite elements [7, 28, 29] and also lattice elements [30].

More recently, these problems had started to be used as a benchmark for testing new methodologies aiming at modelling crack propagation in three dimensions [31-35]. Here, in order to have data for comparison, we choose the geometry considered in [7, 31-35], consisting of a $40 \mathrm{~mm}$ thick steel disc, with radius equal to $200 \mathrm{~mm}$, and a concrete cylindrical block with radius and thickness of $700 \mathrm{~mm}$ and $600 \mathrm{~mm}$, respectively.

As shown in Figure 16, the problem is axis-symmetric, so by applying symmetry conditions in the lateral vertical faces just a quarter of the specimen needs to be modeled. The anchor bolt was not explicitly modeled in the simulation, so the vertical load $\mathrm{F}$ was applied directly in the steel disc, being the concrete block pulled against a counter-pressure ring placed on the superior surface of the block and fixed in the vertical direction. The steel disc was assumed connected to the concrete only at top face, whereas no contact has been assumed along the bottom and lateral surfaces.

The numerical analysis was held by using arch-length techniques so eventual snap-backs exhibited by the response can be traced. 


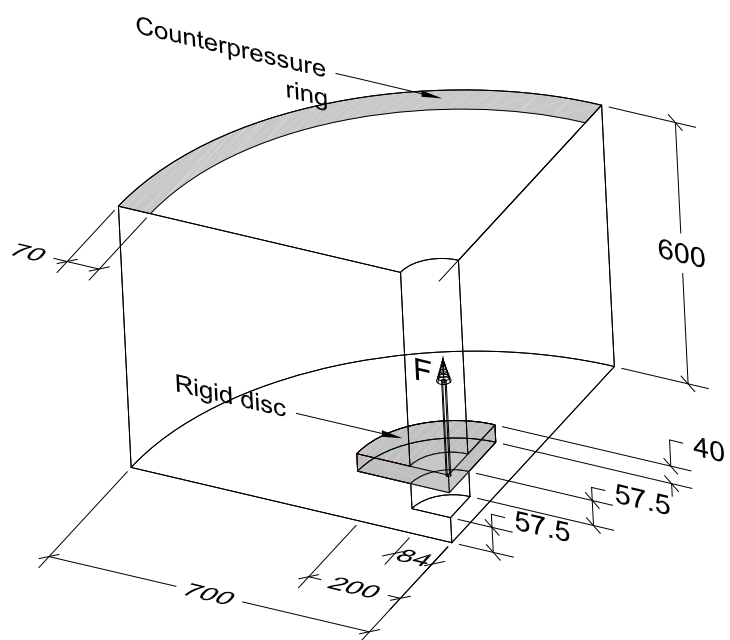

\begin{tabular}{cccc:c}
\hline $\begin{array}{c}\sigma_{u} \\
{[\mathrm{MPa}]}\end{array}\left[\begin{array}{c}{[\mathrm{GPa}]} \\
{[\mathrm{N}}\end{array}\right.$ & $v$ & $\begin{array}{c}G_{f} \\
{[\mathrm{kN} / \mathrm{m}]}\end{array}$ & $\gamma$ \\
\hline 3.0 & 30.0 & 0.2 & 0.1 & 0.95 \\
\hline
\end{tabular}

Figure 16: Pull out test. Geometry $(\mathrm{mm})$ and material parameters, being $s_{u}$ the ultimate stress, $E$ the Young's modulus, $n$ the Poisson's ratio, $G_{f}$ the fracture energy and $g$ is the user-parameter defining the DDM injection threshold (see REMARK 3-1).

In the analysis presented in [7] the author identified two different mechanisms of failure: i) a splitting mode and ii) a conical pull-out failure mode. Here we are interested in simulating the conical pull-out failure where the crack propagates from the upper circular edge of the steel disk into the concrete block towards the counter-pressure ring.

Figure 17 shows the evolution of the pull-out failure mechanism simulated by using the strain injection techniques and a Rankine plasticity constitutive model, for three representative stages of analysis.

A

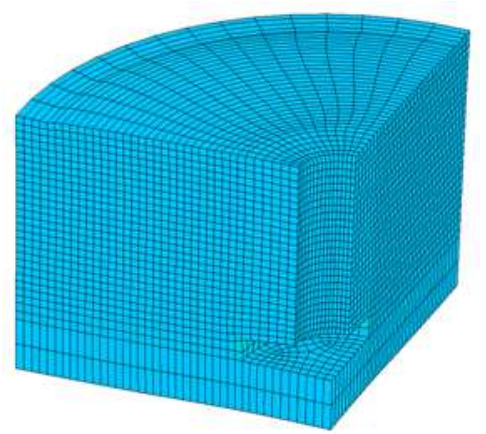

B

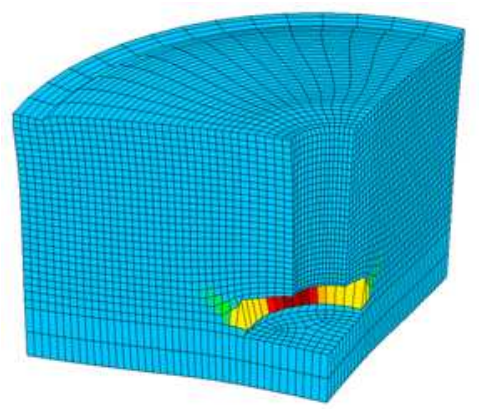

$\mathrm{C}$

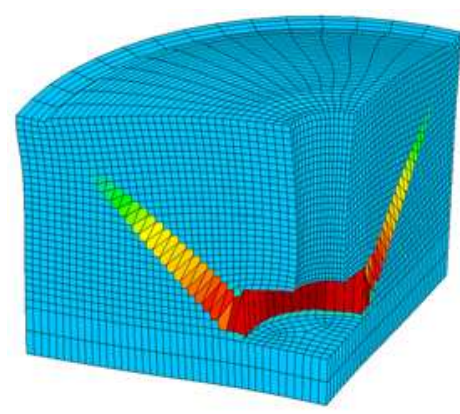

Figure 17: Pull out test. Deformed mesh and equivalent plastic deformations for three stages of analysis (the stages are indicated in the force displacement curve depicted in Figure 18Figure 18). The finite element mesh has a total of 16144 finite elements and 48432 degrees of freedom.

In Figures 18 and 19 the results obtained with the strain injection techniques are compared with those computed with the standard displacement-based finite element formulation. In Figure 18, a quantitative comparison, in terms of the force displacement curves, is presented, while in Figure 19 the equivalent plastic deformations at the final stages of analysis are plotted, this allowing to compare qualitatively the distribution of the non-linear effects. This latter figure shows that the results obtained using a standard displacement-based finite element formulation, suffer from severe stress locking and the pullout failure mechanism does not evolve. In contrast, it can be observed that, by using the strain injection techniques. The conical pull-out mechanism is formed and that the non-linear phenomena concentrate 
in a band encompassing just one finite element, this indicating that stress locking effects are much circumvented [36].

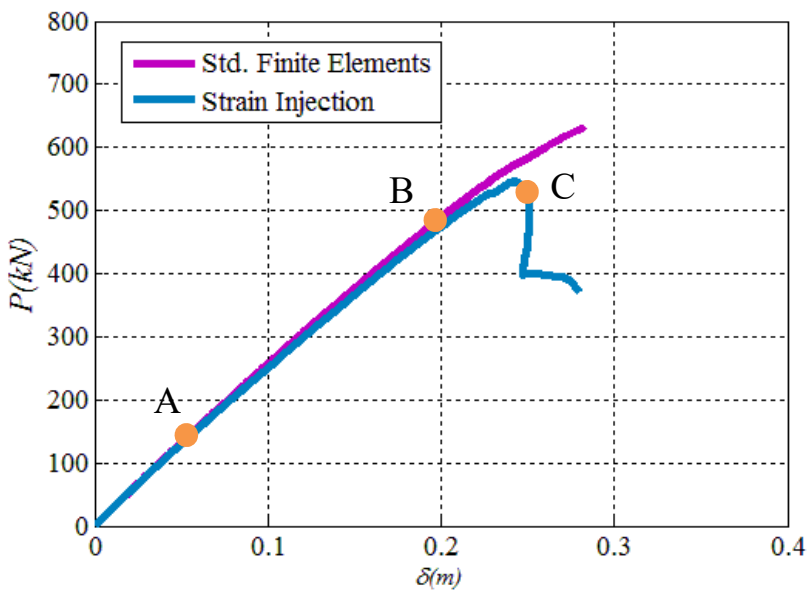

Figure 18: Pull out test. Force displacement curves.

(The points in the curve correspond to the 3 stages in Figure 17).

a)

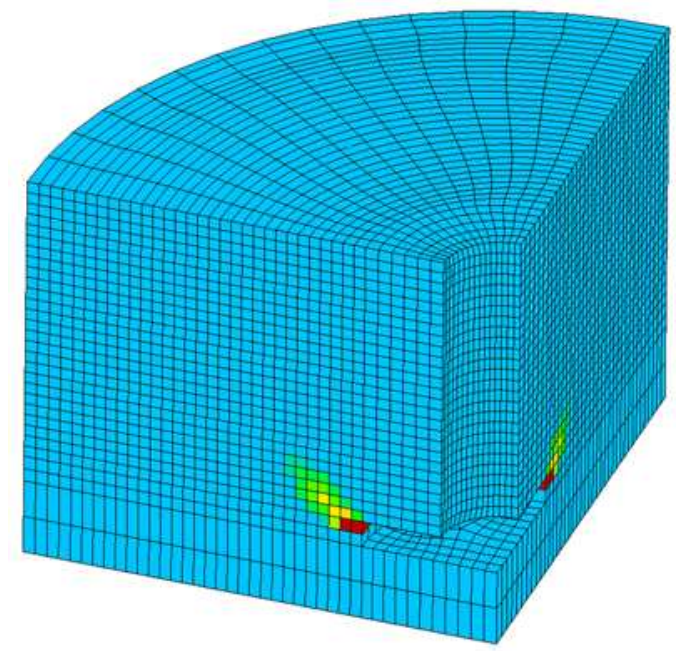

b)

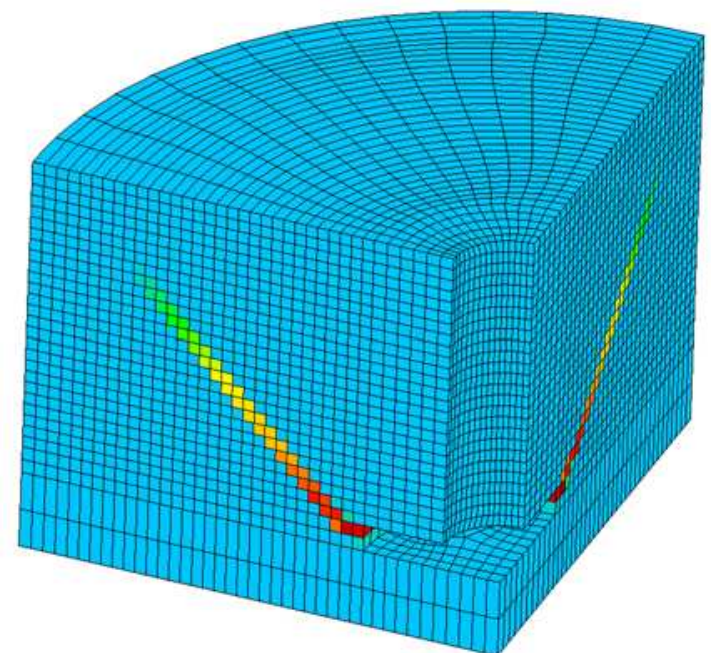

Figure 19: Pull out test. Equivalent plastic deformations for the final stage of analysis: a) Standard displacement-based finite element formulation and b) Strain injection-based technique.

In Figure 20 some additional results obtained with the strain injection techniques are presented: the crack path field and a smooth field of the equivalent plastic deformations (depicted now by means of iso-surfaces, so that a three dimensional perspective can be provided). Both figures show the conical shape of the failure mechanism. 
a)

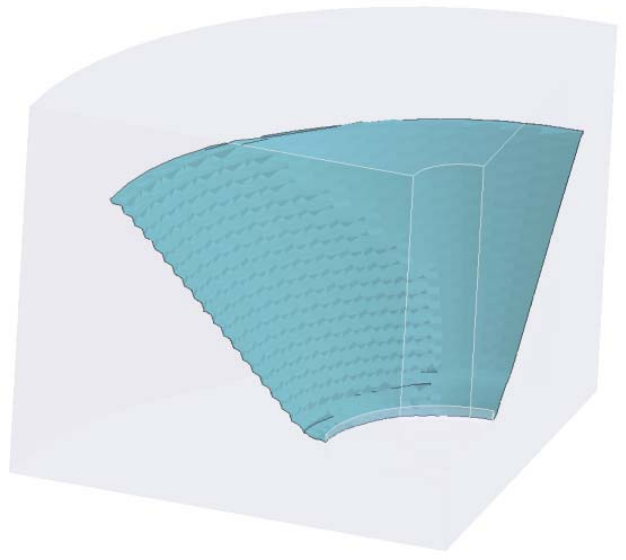

b)

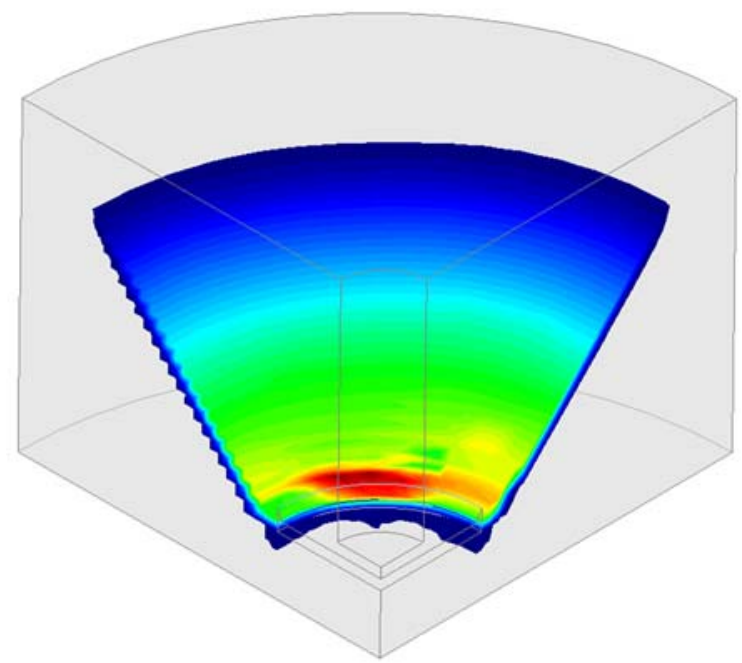

Figure 20: Pull out test. Results obtained with the strain injection techniques: a) Crack path field and b) iso-surfaces of the equivalent plastic deformation (smothed).

In Figure 21 the results obtained with the strain injection techniques are compared with results obtained by other authors. In terms of the crack trajectory of the pull out mechanism the various solutions are quite similar, i.e. the crack propagates from the upper circular edge of the steel disk at the bottom of the concrete block towards the counter pressure ring.

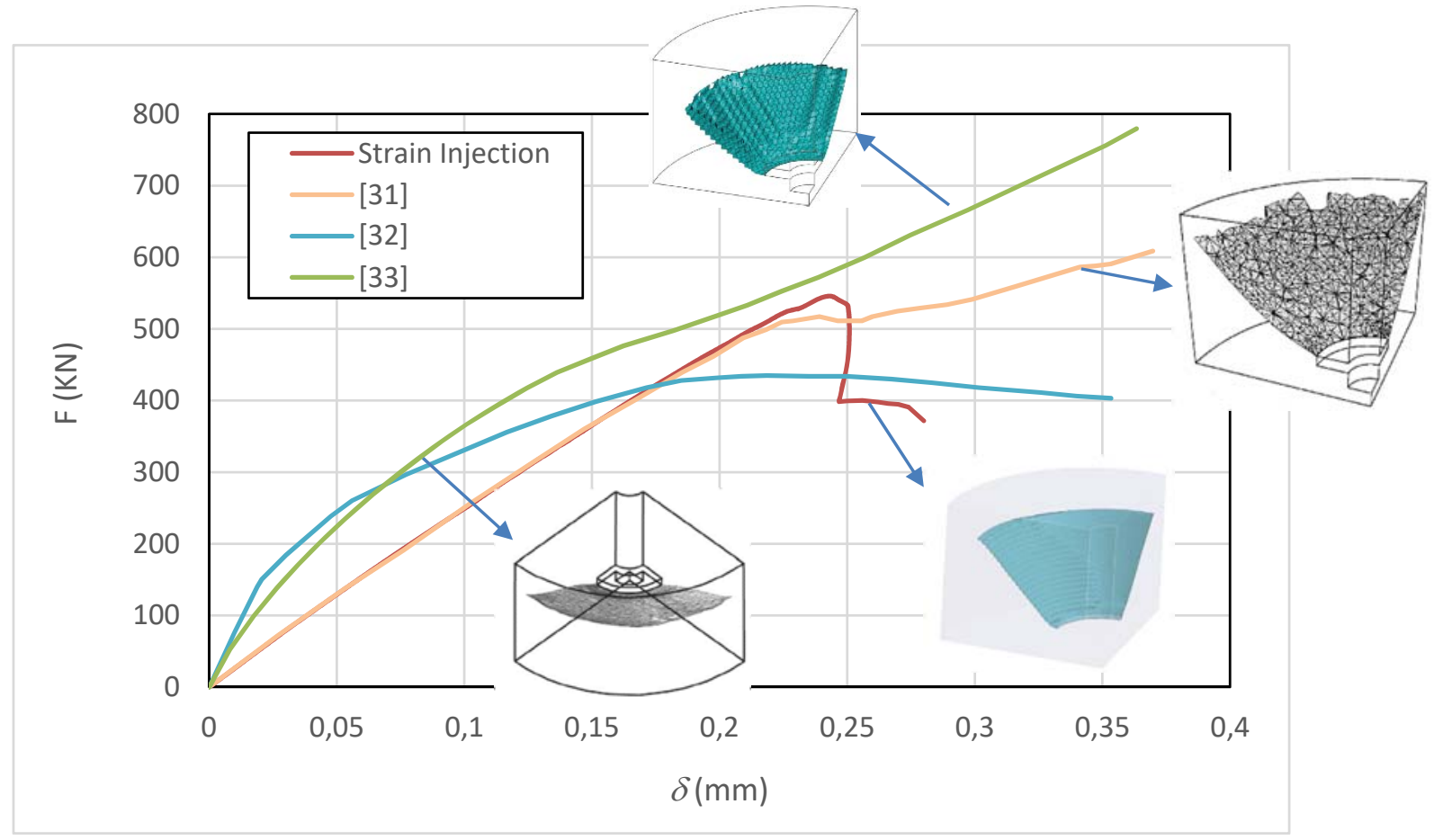

Figure 21: Pull out test. Force displacement curves. Comparison with other authors.

As for the quantitative results (force-displacement curves in Figure 21) some relevant differences are found. In fact, the solutions provided in [32] (using X-FEM) and [33] (using stabilized mixed formulations) are more stiffer throughout the loading process. On the contrary, the results in [31], using a partition of unity method (PUM), and the ones obtained with the techniques proposed in this work, both using the concept of "embedded discontinuities", display a more flexible response and both lead 
to very similar peak loads. However, it is worth mentioning that the only result exhibiting structural softening is the one obtained with the strain injection techniques proposed in this work.

\subsection{Brokenshire torsion test}

This torsion test was proposed by Brokenshire [37] for the experimental study of fracture torsion and consists of a prismatic concrete beam with a notch inclined by $45^{\circ}$ in relation to the top and down surfaces. The experimental setup used by Brokenshire is depicted in Figure 22, where it is shown that the beam is restrained by two steel frames at both ends, being supported at three corners and loaded at the fourth corner, this generating the torsional moment.

The Brokenshire torsion test has attracted large attention from the computational mechanics community [38-46] since the experimentally reported results, consisting of a nonplanar curved crack trajectory (Figure 23), are challenging for those numerical methods aiming to simulate 3D nonplanar crack propagation. Here we are presenting some numerical results obtaining by using the strain injection techniques and a Rankine plasticity model. Again, as for the four-point bending test in section 5.2, for releasing the kinematic locking, that arises when Rankine-type plasticity models and strong discontinuities are used, the regularization parameter $k$, in equation (10), was set equal to the elemental effective bandwidth $1^{(e)}$ (see REMARK 5-1).

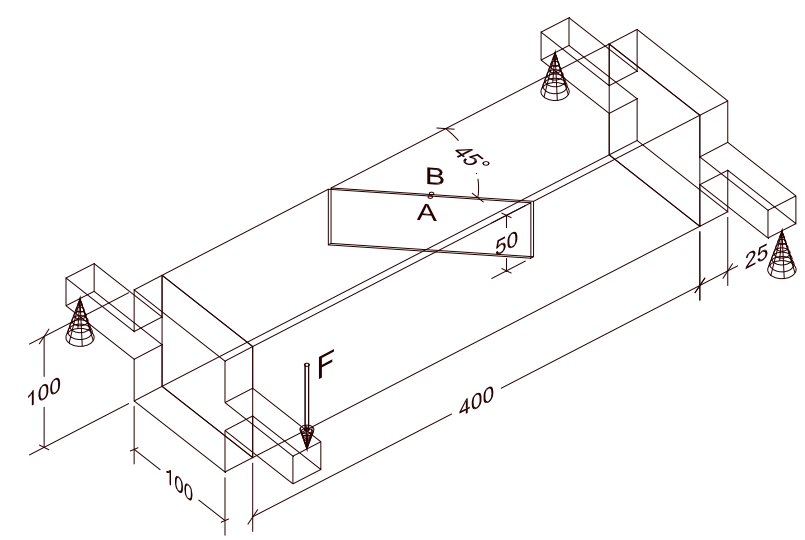

\begin{tabular}{cccc:c}
\hline $\begin{array}{c}\sigma_{u} \\
{[\mathrm{MPa}]}\end{array}\left[\begin{array}{c}{[\mathrm{GPa}]} \\
{[2.3}\end{array}\right.$ & $v$ & $\begin{array}{c}G_{f} \\
{[\mathrm{kN} / \mathrm{m}]}\end{array}$ & $\gamma$ \\
\hline 2.0 & 0.2 & 0.08 & 0.9 \\
\hline
\end{tabular}

Figure 22: Brokenshire torsion test. Geometry $(\mathrm{mm})$ and material parameters, being $s_{u}$ the ultimate stress, $E$ the Young's modulus, $n$ the Poisson's ratio, $G_{f}$ the fracture energy and $g$ is the user-parameter defining the DDM injection threshold (see REMARK 3-1). 


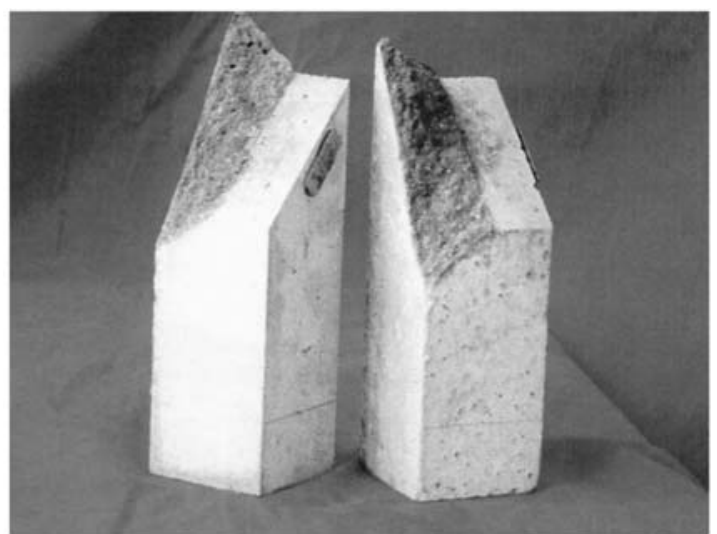

Figure 23: Brokenshire torsion test. Experimentally obtained crack surface. Adapted from [38].

In Figure 24 the finite element mesh used in the simulations and a curve displaying the applied force versus the Crack Mouth Opening Displacement (CMOD), between points A and B (of Figure 22-a). are depicted. In turn, Figure 25 shows the evolution of the injection domain, at the front surface, for three representative stages of loading (which are signaled in Figure 24-b), while Figure 26 shows a three dimensional view of the evolution of the crack path at the same stages of loading. In this latter figure it can be appreciated a nonplanar curved crack, propagating downwards from the notch tip, that compares well with the experimentally reported results (shown in Figure 23). The crack rotation in the horizontal plane can be better appreciated in Figure 27, which shows a top view of the crack path. In Figure 28 are shown the iso-surfaces of equivalent plastic deformation and an amplified deformed mesh. In these figures, it can be observed that the non-linear phenomena and the displacement jump are captured in a sharp band encompassing just one finite element.

a)

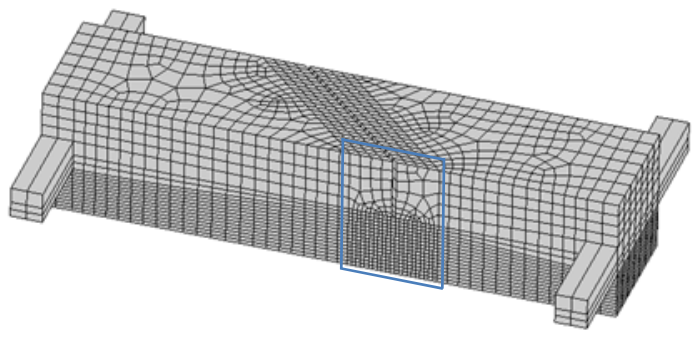

b)

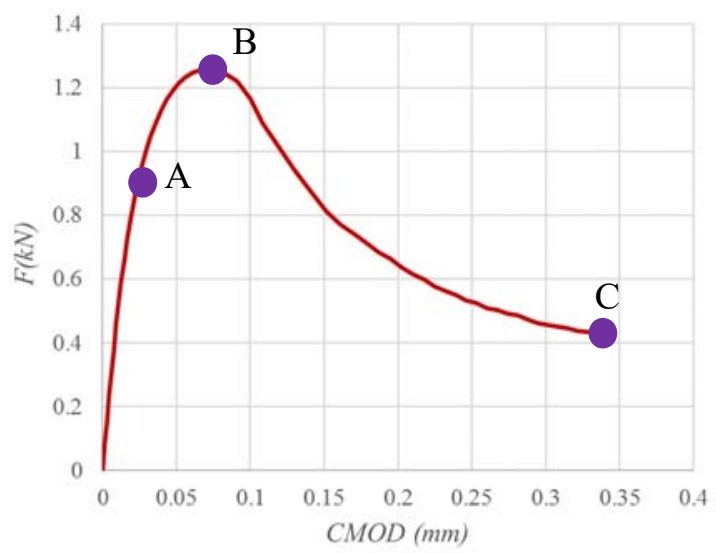

Figure 24: Brokenshire torsion test: a) Finite element mesh and b) Force vs CMOD curve. The finite element mesh has a total of 15230 finite elements and 52929 degrees of freedom. 
A

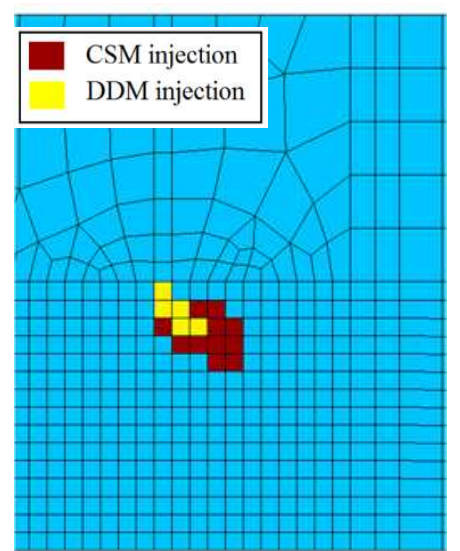

Figure 25: Brokenshire torsion test. Evolution of the injection domain for three stages of loading at the lateral surface of the beam.

A

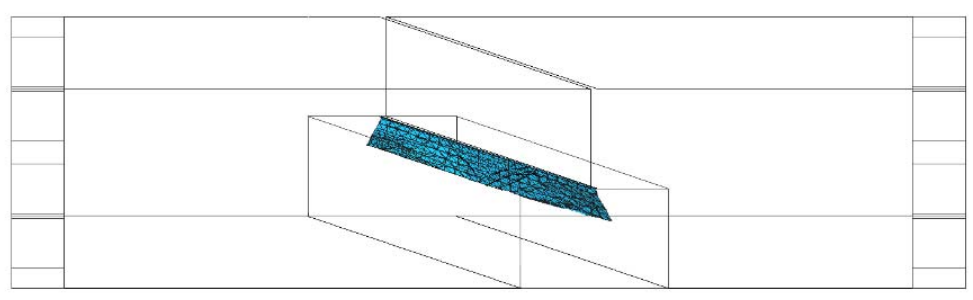

B
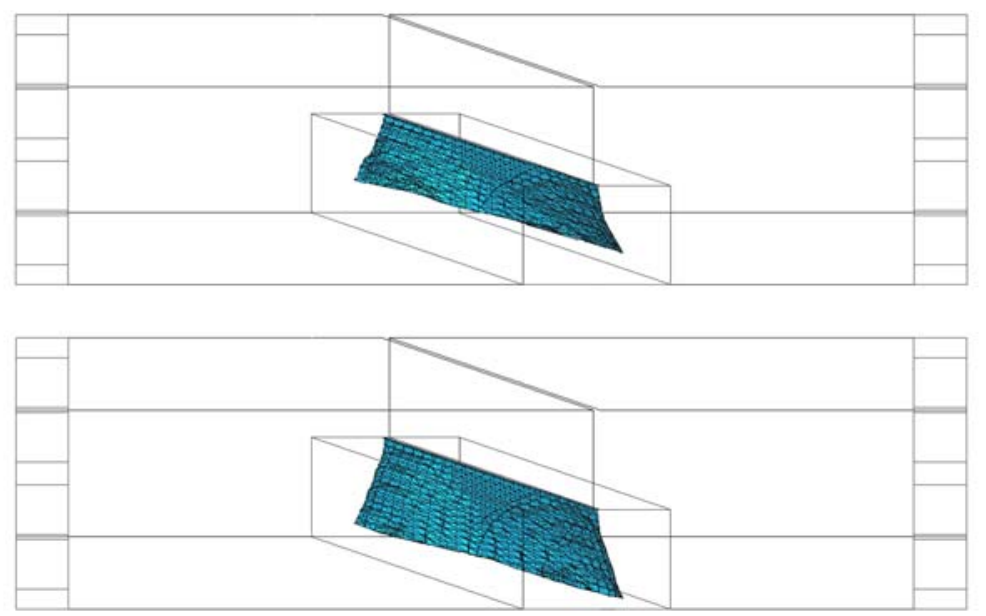

Figure 26: Brokenshire torsion test. Evolution of the crack path at different stages of the analysis. 


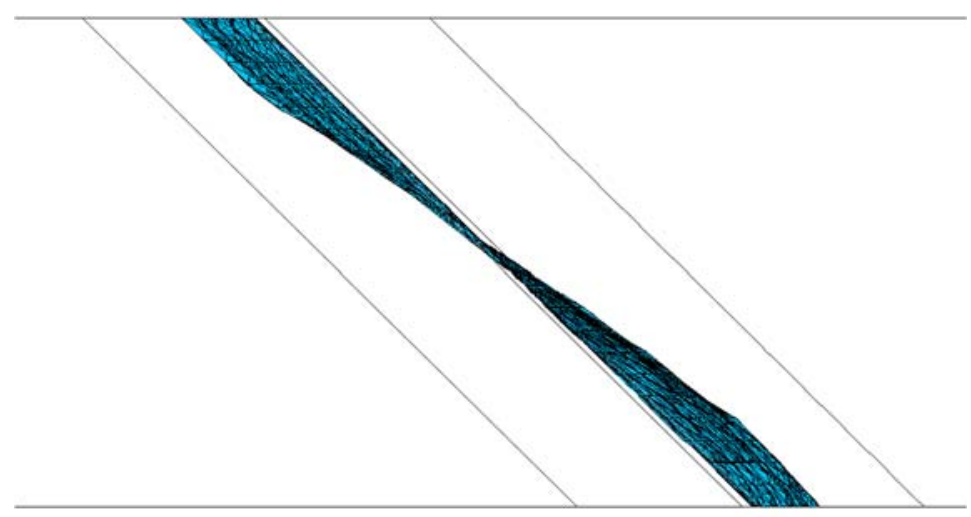

Figure 27: Brokenshire torsion test. Top view of the Crack path.

a)

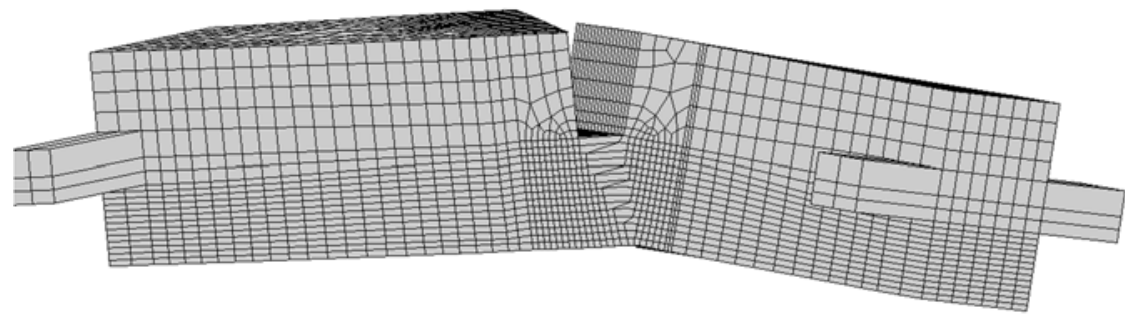

b)

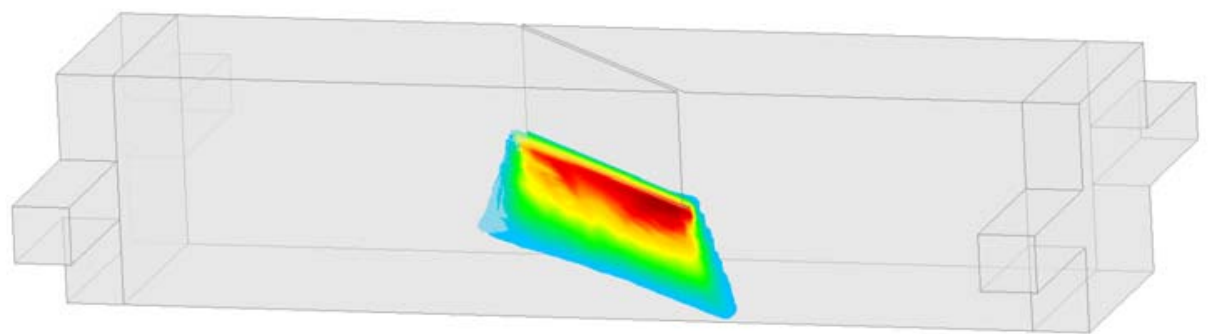

Figure 28: Brokenshire torsion test. Results obtained with the strain injection techniques: a) amplified deformed mesh; b) iso-surfaces of equivalent plastic deformation.

In Figure 29 the force-displacement curves obtained with the strain injection techniques are compared with the ones obtained experimentally by Brokenshire. These comparisons involve the applied force versus the crack mouth opening displacement (CMOD) and also the applied force versus the crack mouth sliding displacement (CMSD). It is observed that the strain injection solution captures well the limit load as well as the post-limit behavior. 
a)

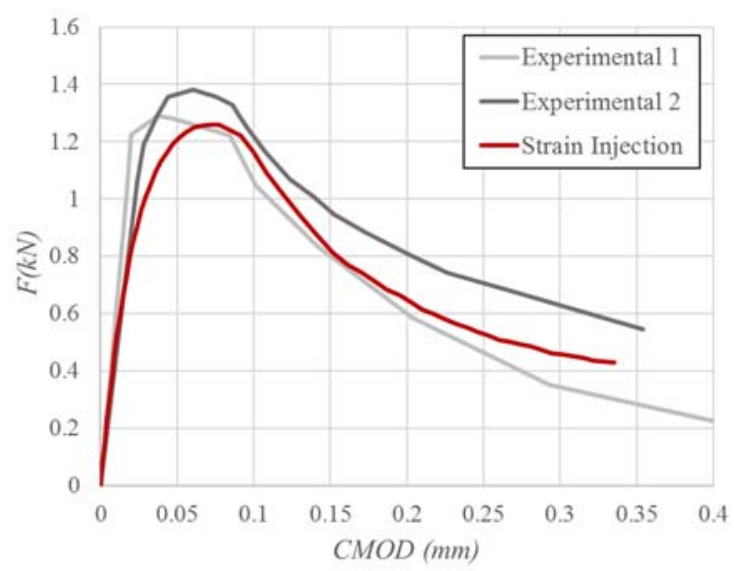

b)

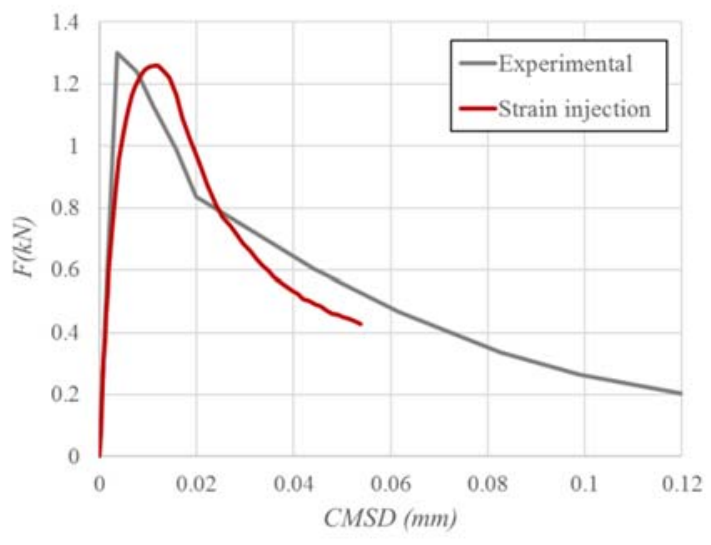

Figure 29: Brokenshire torsion test: a) Force-CMOD curve and b) Force-CMSD curve.

\section{Conclusions}

This paper presents a natural extension to real-life three dimensional cases of the crack path field and strain injection techniques, that have been proposed by the authors in previous publications and assessed in ideal 2D problems [1-4]. The numerical benchmarks clearly show the applicability of the proposed techniques for modeling complex crack trajectories in three dimensions.

The advantages of the methodology, pointed in previous publications, are retained by the present threedimensional implementation. In this sense, from this work it might be concluded the following for the $3 \mathrm{D}$ version of the proposed approach:

- As shown in section 5, where representative numerical applications were presented, coarse finite element meshes can be used. Besides, the additional degrees of freedom, for injecting the discontinuous modes, are condensed out at the element level, this meaning that the computational cost of the methodology remains almost the same than using standard displacement-based finite element formulations.

- The obtained results also provided evidence that stress-locking effects are much minimized and that the three-dimensional crack trajectories are independent of the mesh directions, so that mesh-bias effects are removed.

- In this work, either isotropic damage or a Rankine plasticity have been used to model the fracturing material, but the techniques can be immediately extended to other continuum constitutive models exhibiting strain-softening.

The proposed strain injection techniques can be regarded as a mesh independent time-affordable methodology for modelling $3 D$ crack propagation, which can be used in standard personal computers. The authors think that it can be competitive in front of alternative methodologies, both in terms of accuracy of the obtained results and response-time of the numerical computations. As an illustration, the most relevant computational issues (including the computational cost) involved in the resolution of the 3D examples presented in this paper are presented in Table 1Table 1: Relevant computational features in 3D problems. M. The used coarse meshes required by the proposed approach, translate into very affordable computational times in a regular desktop. 


\begin{tabular}{|c|c|c|c|}
\hline Problem & $\begin{array}{l}\text { Number of } \\
\text { finite elements }\end{array}$ & $\begin{array}{c}\text { Number of degrees } \\
\text { of freedom }\end{array}$ & $\begin{array}{l}\text { Computational } \\
\text { cost (hours) }\end{array}$ \\
\hline $\begin{array}{l}\text { Homogeneous } \\
\text { strip }\end{array}$ & 1464 & 6336 & $0.1 \mathrm{~h}$ \\
\hline $\begin{array}{l}\text { Four-point } \\
\text { bending test }\end{array}$ & 25600 & 83916 & $16 \mathrm{~h}$ \\
\hline Pull-out test & 18424 & 48432 & $6 \mathrm{~h}$ \\
\hline $\begin{array}{l}\text { Brokenshire } \\
\text { torsion test }\end{array}$ & 15230 & 52929 & $6 \mathrm{~h}$ \\
\hline
\end{tabular}

Table 1: Relevant computational features in 3D problems. The computational cost is approximate and depends essentially in the problem dimension and also in the number of time steps used in the computations. Table 1: Relevant computational features in 3D problems. M.

\section{ACKNOWLEDGMENTS}

Ivo Dias gratefully acknowledges the financial support from Laboratório Nacional de Engenharia Civil (LNEC) through the postdoctoral research Grant (CoMatFail project).

Oriol Lloberas-Valls gratefully acknowledges the funding received from the Spanish Ministry of Economy and Competitiveness through the National Research Plan 2014: MAT2014-60919-R.

\section{REFERENCS}

1. Dias, I.F., J. Oliver, and A.E. Huespe, Strain Injection Techniques in Numerical Modeling of Propagating Material Failure, in Monograph CIMNE $N^{o}-134$. 2012, International Center for Numerical Methods in Engineering: Barcelona. http://www.cimne.com/compdesmat/cvdata/cntr1/dtos/img/mdia/Downloads/M134.pdf.

2. Oliver, J., I.F. Dias, and A.E. Huespe, Crack-path field and strain-injection techniques in computational modeling of propagating material failure. Computer Methods in Applied Mechanics and Engineering, 2014. 274(0): p. 289348.http://www.sciencedirect.com/science/article/pii/S0045782514000139

3. Lloberas-Valls, O., A.E. Huespe, J. Oliver, and I.F. Dias, Strain injection techniques in dynamic fracture modeling. Computer Methods in Applied Mechanics and Engineering, 2016. 308: p. 499534.http://www.sciencedirect.com/science/article/pii/S0045782516304248

4. Dias, I.F., J. Oliver, J.V. Lemos, and O. Lloberas-Valls, Modeling tensile crack propagation in concrete gravity dams via crack-path-field and strain injection techniques. Engineering Fracture Mechanics, $2016 . \quad 154$ p. 310.http://www.sciencedirect.com/science/article/pii/S0013794415007146

5. Rashid, Y., Analysis or prestressed concrete pressure vessels. Nuclear Engineering and Design 1968. 7: p. 773-782 
6. Rots, J.G., P. Nauta, G.M.A. Kusters, and J. Blaauwendraad, Smeared crack approach and fracture localization in concrete. Heron, 1985. 30(1): p. 1-49

7. Rots, J.G., Computational Modeling of Concrete Fracture. 1988, Delft University of Technology.

8. Comi, C. and U. Perego, Numerical aspects of nonlocal damage analyses. Revue Européenne des Éléments Finis, 2001. 10(2-4): p. 227242.https://doi.org/10.1080/12506559.2001.11869249

9. Pijaudier Cabot, G. and Z. Bazant, Nonlocal damage theory. Journal Engineering Mechanics ASCE, 1987. 113: p. 1512-1533.http://dx.doi.org/10.1061/(ASCE)07339399(1987)113:10(1512)

10. De Borst, R. and M.A. Guitiérrez, A unified framework for concrete damage and fracture models including size effects. International Journal of Fracture, 1999. 95(1): p. 261277.https://doi.org/10.1023/A:1018664705895

11. de Borst, R. and H.-B. Mühlhaus, Gradient-dependent plasticity: Formulation and algorithmic aspects. International Journal for Numerical Methods in Engineering, 1992. 35(3): p. 521-539.http://dx.doi.org/10.1002/nme.1620350307

12. Nguyen, Q.-S. and S. Andrieux, The non-local generalized standard approach: a consistent gradient theory. Comptes Rendus Mécanique, 2005. 333(2): p. 139145.http://www.sciencedirect.com/science/article/pii/S1631072104002244

13. Hakim, V. and A. Karma, Laws of crack motion and phase-field models of fracture. Journal of the Mechanics and Physics of Solids, 2009. 57(2): p. 342368.http://www.sciencedirect.com/science/article/pii/S0022509608001774

14. Miehe, C., M. Hofacker, and F. Welschinger, A phase field model for rate-independent crack propagation: Robust algorithmic implementation based on operator splits. Computer Methods in Applied Mechanics and Engineering, 2010. 199(45-48): p. 27652778.http://www.sciencedirect.com/science/article/pii/S0045782510001283

15. Simo, J. and J. Oliver. A new approach to the analysis and simulation of strong discontinuities. in Fracture and Damage in Quasi-brittle Structures. 1994: \$ $\backslash$ \& FN Spon.

16. Oliver, J. The strong discontinuity approach: an overview. in WCCM98, IV World Congress on Computational Mechanics. 1998. Buenos Aires, Argentina: CIMNE.

17. Armero, F., Strong discontinuities in antiplane/torsional problems of computational failure mechanics. International Journal of Fracture, 2012. 178(1-2): p. 3-32

18. Belytschko, T., N. Moes, S. Usui, and C. Parimi, Arbitrary discontinuities in finite elements. International Journal for Numerical Methods in Engineering, 2001. 50(4): p. 9931013.http://onlinelibrary.wiley.com/doi/10.1002/1097-

0207(20010210)50:4\%3C993::AID-NME164\%3E3.0.CO;2-M/abstract

19. Sukumar, N., N. Moës, B. Moran, and T. Belytschko, Extended finite element method for three-dimensional crack modelling. International Journal for Numerical Methods in Engineering, 2000. 48(11): p. 1549-1570.http://dx.doi.org/10.1002/10970207(20000820)48:11<1549::AID-NME955>3.0.CO;2-A

20. Lloberas-Valls, O., A.E. Huespe, J. Oliver, and I.F. Dias, Applications of Crack Path Field and Strain Injection Techniques in Dynamic Fracture of Quasi-Brittle Materials, in International Conference on Computational Plasticity. Fundamentals and Applications. COMPLAS XIII, CIMNE, Editor. 2015: Barcelona.

21. Rice, J.R., The Localization of Plastic Deformation, in Theoretical and Applied Mechanics, W.T. Koiter, Editor. 1976, North-Holland Publ. Co. p. 207-220. 
22. Arrea, M. and A.R. Ingraffea, Mixed-mode Crack Propagation in Mortar and Concrete. 1982, Dept. Struct. Eng. Cornell Univ. New York.

23. Oliver, J., A.E. Huespe, M.D.G. Pulido, and E. Chaves, From continuum mechanics to fracture mechanics: the strong discontinuity approach. Engineering Fracture Mechanics, 2002. 69(2): p. 113-136.https://doi.org/10.1016/S0013-7944(01)00060-1

24. Jirasek, M. and P. Grassl, Evaluation of directional mesh bias in concrete fracture simulations using continuum damage models. Engineering Fracture Mechanics, 2008. 75(8): p. 1921-1943

25. Dias-da-Costa, D., J. Alfaiate, L.J. Sluys, and E. Júlio, A comparative study on the modelling of discontinuous fracture by means of enriched nodal and element techniques and interface elements. International Journal of Fracture, 2009. 161(1): p. 97.https://doi.org/10.1007/s10704-009-9432-6

26. Cendón, D.A., J.C. Gálvez, M. Elices, and J. Planas, Modelling the fracture of concrete under mixed loading. International Journal of Fracture, 2000. 103(3): p. 293310.https://doi.org/10.1023/A:1007687025575

27. Oliver, J., M. Cervera, and O. Manzoli, Strong discontinuities and continuum plasticity models: the strong discontinuity approach. International Journal of Plasticity, 1999. 15(3): p. 319-351.<Go to ISI $>$ ://000079597700005

28. de Borst, R., Non-linear analysis of frictional materials. 1986, Ph.D. Thesis, Delft Univ. of. Technology.

29. Ottosen, N.S., Nonlinear Finite Element Analysis of Pull-Out Test. Journal of the Structural Division, ASCE, 1981. 107(4): p. 591-603

30. Vervuurt, A., J.G.M. Van Mier, and E. Schlangen, Analyses of anchor pull-out in concrete. Materials and Structures, 1994. 27(5): p. 251-259.https://doi.org/10.1007/BF02473041

31. Gasser, T.C. and G.A. Holzapfel, Modeling 3D crack propagation in unreinforced concrete using PUFEM. Comp. Meth. in Appl. Mech. Engrg., 2005. 194(25-26): p. 2859-2896

32. Areias, P.M.A. and T. Belytschko, Analysis of three-dimensional crack initiation and propagation using the extended finite element method. International Journal for Numerical Methods in Engineering, 2005. 63(5): p. 760-788.http://dx.doi.org/10.1002/nme.1305

33. Benedetti, L., M. Cervera, and M. Chiumenti, High-fidelity prediction of crack formation in $2 D$ and $3 D$ pullout tests. Computers \& Structures, 2016. 172: p. 93109.http://www.sciencedirect.com/science/article/pii/S0045794916302243

34. Kaczmarczyk, L., M.M. Nezhad, and C. Pearce, Three-dimensional brittle fracture: configurational-force-driven crack propagation. International Journal for Numerical Methods in Engineering, 2014. 97(7): p. 531-550.http://dx.doi.org/10.1002/nme.4603

35. Rabczuk, T., S. Bordas, and G. Zi, A three-dimensional meshfree method for continuous multiple-crack initiation, propagation and junction in statics and dynamics. Computational Mechanics, 2007. 40(3): p. 473-495.https://doi.org/10.1007/s00466-006-0122-1

36. Oliver, J., A.E. Huespe, and I.F. Dias, Strain localization, strong discontinuities and material fracture: Matches and mismatches. Computer Methods in Applied Mechanics and Engineering, 2012. 241-244: p. 323-336

37. Brokenshire, D.R., A study of torsion fracture tests. 1996, Cardiff University: Cardiff

38. Jefferson, A.D., B.I.G. Barr, T. Bennett, and S.C. Hee, Three dimensional finite element simulations of fracture tests using the Craft concrete model. Computers and Concrete, 2004. 1(3): p. 261-284

39. Gasser, T.C. and G.A. Holzapfel, 3D Crack propagation in unreinforced concrete.: A twostep algorithm for tracking 3D crack paths. Computer Methods in Applied Mechanics and 
Engineering,

2006.

195(37):

p.

5198-

5219.http://www.sciencedirect.com/science/article/pii/S0045782505005426

40. Gürses, E. and C. Miehe, $A$ computational framework of three-dimensional configurational-force-driven brittle crack propagation. Computer Methods in Applied Mechanics and Engineering, 2009. 198(15): p. 14131428.http://www.sciencedirect.com/science/article/pii/S0045782508004301

41. Linder, C. and X. Zhang, A marching cubes based failure surface propagation concept for three-dimensional finite elements with non-planar embedded strong discontinuities of higher-order kinematics. International Journal for Numerical Methods in Engineering, 2013. 96(6): p. 339-372

42. Mesgarnejad, A., B. Bourdin, and M.M. Khonsari, Validation simulations for the variational approach to fracture. Computer Methods in Applied Mechanics and Engineering, 2015. 290: p. 420-437

43. Ferté, G., P. Massin, and N. Moës, 3D crack propagation with cohesive elements in the extended finite element method. Computer Methods in Applied Mechanics and Engineering, 2016. 300: p. 347-374

44. Benedetti, L., M. Cervera, and M. Chiumenti, 3D numerical modelling of twisting cracks under bending and torsion of skew notched beams. Engineering Fracture Mechanics, 2017. 176: p. 235-256.http://www.sciencedirect.com/science/article/pii/S0013794417300607

45. Kaczmarczyk, Ł., Z. Ullah, and C.J. Pearce, Energy consistent framework for continuously evolving 3D crack propagation. Computer Methods in Applied Mechanics and Engineering, 2017. 324: p. 54-73

46. Baydoun, M. and T.P. Fries, Crack propagation criteria in three dimensions using the $X F E M$ and an explicit-implicit crack description. International Journal of Fracture, 2012. 178(1): p. 51-70.https://doi.org/10.1007/s10704-012-9762-7 